\title{
Approximate Cloaking for the Helmholtz Equation via Transformation Optics and Consequences for Perfect Cloaking
}

\author{
HOAI-MINH NGUYEN \\ Courant Institute
}

\begin{abstract}
In this paper, we study approximate cloaking of active devices for the Helmholtz equation in the whole space of dimension 2 or 3 using the scheme introduced by Kohn, Shen, Vogelius, and Weinstein. More precisely, we assess the degree of invisibility, determine the limit of the field inside the cloaked and cloaking regions, and show that the scheme is unstable with respect to the material parameters. As a consequence, we obtain some feasible properties of "perfect" cloaking. (C) 2011 Wiley Periodicals, Inc.
\end{abstract}

\section{Introduction}

Cloaking via a change of variables was introduced by Greenleaf, Lassas, and Uhlmann [10] for electrical impedance tomography; Pendry, Schurig, and Smith [22] for the Maxwell system; and Leonhardt [15] in the geometric optics setting. They used a singular change of variables that blows up a point into a cloaked region. This singular structure implies difficulties not only in practice but also in analysis. Some approaches $[5,26,27]$ are proposed to tackle this problem mathematically based on the notion of a "weak" solution. To avoid using the singular structure, regularized schemes have been proposed in [2, 6, 12, 23, 24, 29]. The reader can find more information and references related to cloaking in the works mentioned above or in the reviews [8, 28].

In this paper, we study approximate cloaking of active devices for the Helmholtz equation in the whole space of dimension 2 or 3 for the scheme introduced by Kohn, Shen, Vogelius, and Weinstein in [12], where they used a transformation that blows up a small region instead of a point into the cloaked region. We assess the degree of invisibility, determine the limit of the field inside the cloaked and cloaking regions, and show that the scheme is unstable with respect to the material parameters. As a consequence, we obtain some feasible properties of "perfect" cloaking. More precisely, let $\varepsilon$ denote the parameter of regularization, i.e., the ratio between the diameter of the region blown up to the cloaked region $D$ and the diameter of the cloaked region:

Communications on Pure and Applied Mathematics, Vol. LXV, 0155-0186 (2012)

(C) 2011 Wiley Periodicals, Inc. 
(1) In the three-dimensional nonresonant case, i.e., when the frequency is not an eigenvalue of the Neumann problem in $D$, we show that the difference between the field and the "push-forward" of the solution of the Helmholtz equation in free space is of order $\varepsilon$ in any bounded region away from $D$ (Theorem 1.4). Concerning the limiting behavior of the approximate field in $D$, we prove that it converges and the limit is the solution of the corresponding Neumann problem in $D$ (Theorem 1.4 and Definition 1.5).

(2) In the three-dimensional resonant case, i.e., when the frequency is an eigenvalue of the Neumann problem in $D$, under the assumption that the source inside the cloaked region is orthogonal, with respect to the $L^{2}$-scalar product in $D$, to all Neumann eigenfunctions (this holds if the cloaked region is passive), we establish the following results (Theorem 1.4 and Definition 1.5). First, the difference between the field and the push-forward of the solution of the Helmholtz equation in free space is of order $\varepsilon$ in any bounded region away from $D$. Second, the approximate field converges in $D$, and the limit is uniquely determined. Nevertheless, this limit is not only a solution of the Neumann problem in $D$ but also depends on the value of the solution of the Helmholtz equation in free space at the point where the maps are blown up in a quite involved manner.

(3) In the three-dimensional resonant case, without the assumption on the orthogonality of the source mentioned in statement (2), the energy of the field inside $D$ explodes as $\varepsilon \rightarrow 0$; moreover, cloaking cannot be achieved (Proposition 1.11).

(4) In the two-dimensional nonresonant case (see Definition 1.7), we show that the field converges to the push-forward of the solution of the Helmholtz equation in free space in any bounded set away from $D$ with a rate $1 /|\ln \varepsilon|$, and the limiting behavior of the field in $D$ exhibits a nonlocal structure. Therefore, the limit is generally not the solution of the Neumann problem in $D$ (Theorem 1.8 and Definition 1.7).

(5) In the two-dimensional resonant case (see Definition 1.7), we prove that the energy inside the cloaked region can go to infinity and cloaking cannot be achieved (Proposition 1.12).

(6) Concerning the stability with respect to the material parameters inside the cloaked region without a source, we show that cloaking is unstable with respect to these parameters. Roughly speaking, there exist some fixed parameters such that for each $\varepsilon$, one can perturb these parameters by an amount of order $\varepsilon$ in three dimensions and $1 /|\ln \varepsilon|$ in two dimensions so that the degree of visibility is of order 1 (Proposition 1.13).

Remark 1.1. Property (6) does not contradict Properties (1) and (4) since Property (6) is only stated under a condition on the amount of the perturbation that does not hold when the material parameters are fixed. 
Remark 1.2. A similar conclusion as in Property (6) for two-dimensional bounded domains was previously observed by Kohn et al. [11].

Our results in the three-dimensional nonresonant case are compatible with what has been mentioned in the literature: cloaking is achieved, the energy of the field is finite in the cloaked region, and the limit of the field inside the cloaked region is the solution of the Neumann problem; see, e.g., [5, 26]. However, our results in the three-dimensional resonant case are quite different. In the setting in [5], the limit field can be any Neumann eigenfunction in the cloaked region for a passive device. In [26], the author asserted that the fields inside and outside the cloaked region are completely decoupled from each other. It is discussed in the literature that cloaking for the Helmholtz equation is achieved and the energy of the field inside the cloaked region is finite (see, e.g., [4, 5, 26, 27]). Two-dimensional perfect cloaking has not been studied as extensively as in the three-dimensional case, and it is often argued that the field in the cloaked region is a solution of the Neumann problem.

We recall that the weak solution considered in [5] is only discussed in three dimensions. The degree of invisibility in the approximate cloaking problem is more widely understood when one uses an appropriate lossy layer. In this case, the same estimates as above hold independently of the material parameters in $D$ (see $[11,18,20])$, and there are explicit frequency-dependent estimates that are valid for all frequencies (see [20]). The zero-frequency case is less complicated. This is studied in [12] (see also [19]) where no lossy layer is used and better estimates are obtained. Without a lossy layer, the degree of invisibility in two dimensions is discussed in [23] when the material parameters inside the cloaked region are isotropic and homogeneous, and the approximate cloaking is confirmed for the three-dimensional nonresonant case in [7] (without an estimate of the degree of invisibility). Recently Greenleaf, Kurulev, Lassas, and Uhlmann [9] observed that cloaking without shielding is possible (compare this finding with our results in the three-dimensional resonant case).

Let us describe the problem more precisely. To illustrate the idea, let us suppose that the cloaking region is the annular $\{1<|x|<2\}$ and the cloaked region is the unit ball $B$ centered at the origin of $\mathbb{R}^{d}(d=2,3)$. Using the scheme in [12], the parameters in the cloaking region are given by

$$
a_{c}, \sigma_{c}=F_{\varepsilon *} I, F_{\varepsilon *} 1,
$$

where $F_{\varepsilon}$ is the map that blows up the ball $B_{\varepsilon}$ into $B_{1}$ given by

$$
F_{\varepsilon}= \begin{cases}x & \text { if } x \in \mathbb{R}^{d} \backslash B_{2} \\ \left(\frac{2-2 \varepsilon}{2-\varepsilon}+\frac{|x|}{2-\varepsilon}\right) \frac{x}{|x|} & \text { if } x \in B_{2} \backslash B_{\varepsilon}, \\ \frac{x}{\varepsilon} & \text { if } x \in B_{\varepsilon}\end{cases}
$$


Hereafter we use the standard notation

$$
\begin{gathered}
F_{*} A(y)=\frac{D F(x) A(x) D F^{\top}(x)}{\operatorname{det} D F(x)}, \quad F_{*} \Sigma(y)=\frac{\Sigma(x)}{\operatorname{det} D F(x)}, \\
\text { where } x=F^{-1}(y)
\end{gathered}
$$

for any matrix-valued function $A$ and any complex function $\Sigma$, and we denote by $D_{r}$ the set $\{r x: x \in D\}$ for any open bounded set $D$ of $\mathbb{R}^{d}$ and for any $r>0$.

Let $a$ be a uniformly elliptic matrix-valued function defined in $B_{1}$, and let $\sigma$ be a complex function defined in $B_{1}, f \in L^{2}\left(\mathbb{R}^{d}\right)$, such that $0 \leq \operatorname{essinf} \Im(\sigma) \leq$ $\operatorname{ess} \sup \Im(\sigma)<+\infty, 0<\operatorname{essinf} \Re(\sigma)<\operatorname{ess} \sup \Re(\sigma)<+\infty$, and $\operatorname{supp} f \subset$ $\left(B_{4} \backslash B_{3}\right) \cup \overline{B_{1}}$. For $k>0$, let $u, u_{c} \in H_{\mathrm{loc}}^{1}\left(\mathbb{R}^{d}\right)$ be the unique outgoing solutions of the equations

$$
\Delta u+k^{2} u=f \chi_{\{|x| \geq 2\}} \quad \text { in } \mathbb{R}^{d}
$$

and

$$
\operatorname{div}\left(A_{c} \nabla u_{c}\right)+k^{2} \Sigma_{c} u_{c}=f \quad \text { in } \mathbb{R}^{d},
$$

where $\chi_{\Omega}$ denotes the characteristic function of the set $\Omega$ for any $\Omega \subset \mathbb{R}^{d}$, and

$$
A_{c}, \Sigma_{c}= \begin{cases}I, 1 & \text { in } \mathbb{R}^{d} \backslash B_{2}, \\ a_{c}=F_{\varepsilon *} I, \sigma_{c}=F_{\varepsilon *} 1 & \text { in } B_{2} \backslash B_{1}, \\ a, \sigma & \text { in } B_{1} .\end{cases}
$$

In the following, whenever we talk about outgoing solutions to a Helmholtz problem at frequency $k$, we mean solutions that satisfy

$$
\frac{\partial v}{\partial r}-i k v=o\left(r^{-\frac{d-1}{2}}\right) \quad \text { as } r=|x| \rightarrow \infty,
$$

where $d=2$ or 3 is the dimension of space.

Remark 1.3. Physically $a$ and $\sigma$ are the material parameters in the cloaked region $B_{1} ; f$ is a given source outside the cloaking region, $u$ describes the field corresponding to the source existing outside the cloaked and cloaking regions in free space, and $u_{c}$ describes the field due to the source $f$ in the presence of the cloaking device and the cloaked object.

The effectiveness of the approximate cloak and the limit of $u_{c}$ as $\varepsilon \rightarrow 0$ are described in Theorems 1.4 and 1.8 below. The proofs will be presented in Sections 2 and 3.

TheOREM 1.4. Let $d=3, k>0$, and $0<\varepsilon<1$. Define $e^{1}$

$$
\begin{array}{r}
M:=\left\{\psi \in H^{1}\left(B_{1}\right): \operatorname{div}(a \nabla \psi)+k^{2} \sigma \psi=0 \text { in } B_{1}\right. \\
\text { and } \left.a \nabla \psi \cdot \eta=0 \text { on } \partial B_{1}\right\} .
\end{array}
$$

\footnotetext{
${ }^{1}$ In the following, $\eta$ denotes the unit normal vector on $\partial D$ directed to the complement of $D$ for any smooth bounded open subset $D$ of $\mathbb{R}^{d}$.
} 
In the resonant case, i.e., when $M \neq\{0\}$, assume in addition that $\int_{B_{1}} f \overline{\mathbf{e}}=0$ for any e $\in M .^{2}$ Then for all $K \Subset \mathbb{R}^{3} \backslash \bar{B}_{1}$,

$$
\left\|u_{c}-u \circ F_{0}^{-1}\right\|_{H^{1}(K)} \leq C \varepsilon\|f\|_{L^{2}}
$$

for some positive constant $C$ depending on $k, K$, a, and $\sigma$, but independent of $\varepsilon$ and $f$. We also have that $u_{c} \rightarrow C l_{3}(f)$ weakly in $H^{1}\left(B_{1}\right)$. As a consequence,

$$
\lim _{\varepsilon \rightarrow 0}\left\|u_{c}-C l_{3}(f)\right\|_{L^{2}\left(B_{1}\right)}=0 .
$$

Here and in the following, $F_{0}(x):=\lim _{\varepsilon \rightarrow F_{\varepsilon}}(x)$ for $x \in \mathbb{R}^{d} \backslash\{0\}$ and for $d=2,3$.

Definition 1.5. Let $d=3$. We define $\mathrm{Cl}_{3}$ as follows:

(i) The nonresonant case: $M=\{0\}$. Define $C l_{3}(f)=v$ where $v \in H^{1}\left(B_{1}\right)$ is the unique solution of the system

$$
\begin{cases}\operatorname{div}(a \nabla v)+k^{2} \sigma v=f & \text { in } B_{1}, \\ \left.a \nabla v \cdot \eta\right|_{\text {int }}=0 & \text { on } \partial B_{1} .\end{cases}
$$

(ii) The resonant case: $M \neq\{0\}$. Assume in addition that $\int_{B_{1}} f \overline{\mathbf{e}}=0$ for any e $\in M$. Consider the triple of functions $\left(v_{\text {ext }}, v_{\text {int }}, w\right) \in W^{1}\left(\mathbb{R}^{3} \backslash \bar{B}_{1}\right) \times$ $H^{1}\left(B_{1}\right) \times M^{\perp}$, which is the unique solution of the systems

$$
\begin{cases}\Delta v_{\text {ext }}=0 & \text { in } \mathbb{R}^{3} \backslash \bar{B}_{1}, \\ \operatorname{div}\left(a \nabla v_{\text {int }}\right)+k^{2} \sigma v_{\text {int }}=f & \text { in } B_{1}, \\ \operatorname{div}(a \nabla w)+k^{2} \sigma w=0 & \text { in } B_{1},\end{cases}
$$

and

$$
\begin{cases}v_{\text {ext }}-v_{\text {int }}=-u(0) & \text { on } \partial B_{1} \\ a \nabla v_{\text {int }} \cdot \eta=0 & \text { on } \partial B_{1} \\ \frac{\partial v_{\text {ext }}}{\partial \eta}=a \nabla w \cdot \eta & \text { on } \partial B_{1}\end{cases}
$$

Define

$$
C l_{3}(f)=v_{\text {int }} \text {. }
$$

Hereafter, for a connected smooth open region $U$ of $\mathbb{R}^{3}$ with a bounded complement (this includes $U=\mathbb{R}^{3}$ ), $W^{1}(U)$ is defined as follows: ${ }^{3}$

$$
W^{1}(U)=\left\{\psi \in L_{\mathrm{loc}}^{1}(U): \frac{\psi(x)}{\sqrt{1+|x|^{2}}} \in L^{2}(U) \text { and } \nabla \psi \in L^{2}(U)\right\} .
$$

\footnotetext{
${ }^{2}$ This condition is necessary and sufficient to have a solution $v \in H^{1}\left(B_{1}\right)$ of the system $\operatorname{div}(a \nabla v)+k^{2} \sigma v=f$ in $B_{1}$ and $a \nabla v \cdot \eta=0$ on $\partial B_{1}$ by the Fredholm alternative theory; see, e.g., $[1,14]$.

${ }^{3}$ The space $W^{1}$, which is defined here in three dimensions and later in two dimensions (see Definition 1.7), has been used in the study of the Laplace equation in an unbounded domain, e.g., [17].
} 
On the boundary $\partial D$ of any bounded open set $D \subset \mathbb{R}^{3},\left.\phi\right|_{\text {ext }}$ and $\left.\phi\right|_{\text {int }}$ denote the trace of $\phi$ from the outside and the inside. For any closed subspace $M$ of $H^{1}(D)$, we also denote the space $M^{\perp}$ by

$$
M^{\perp}=\left\{\psi \in H^{1}(D): \int_{D}(\nabla \psi \nabla \bar{\phi}+\psi \bar{\phi}) d x=0 \quad \forall \phi \in M\right\} .
$$

Remark 1.6. The uniqueness of the triple $\left(v_{\text {ext }}, v_{\text {int }}, w\right)$ is established in Lemma 2.3 (Section 2). The existence of $\left(v_{\text {ext }}, v_{\text {int }}, w\right)$ will follow from the proof of Theorem 1.4.

The following definition will be used in Theorem 1.8 concerning the two-dimensional setting.

DEFINITION 1.7. In the two-dimensional setting, the system is nonresonant if the problem

$$
\begin{cases}\Delta w=0 & \text { in } \mathbb{R}^{2} \backslash B_{1}, \\ \operatorname{div}(a \nabla w)+k^{2} \sigma w=0 & \text { in } B_{1}, \\ \left.\frac{\partial w}{\partial \eta}\right|_{\text {ext }}=\left.a \nabla w \cdot \eta\right|_{\text {int }} & \text { on } \partial B_{1} .\end{cases}
$$

only has the zero solution in $W^{1}\left(\mathbb{R}^{2}\right)$. Otherwise, the system is resonant.

In the nonresonant case, we define $C L_{2}(f)=v$ to be the unique solution in $W^{1}\left(\mathbb{R}^{2}\right)$ of the system ${ }^{4}$

$$
\begin{cases}\Delta v=0 & \text { in } \mathbb{R}^{2} \backslash B_{1}, \\ \operatorname{div}(a \nabla v)+k^{2} \sigma v=f & \text { in } B_{1}, \\ \left.\frac{\partial v}{\partial \eta}\right|_{\mathrm{ext}}=\left.a \nabla v \cdot \eta\right|_{\mathrm{int}} & \text { on } \partial B_{1} .\end{cases}
$$

Hereafter, for a connected smooth open region $U$ of $\mathbb{R}^{2}$ with a bounded complement (this include $U=\mathbb{R}^{2}$ ), $W^{1}(U)$ is defined as follows:

$$
\begin{aligned}
& W^{1}(U)= \\
& \quad\left\{\psi \in L_{\mathrm{loc}}^{1}(U): \frac{\psi(x)}{\ln (2+|x|) \sqrt{1+|x|^{2}}} \in L^{2}(U) \text { and } \nabla \psi \in L^{2}(U)\right\} .
\end{aligned}
$$

THEOREM 1.8. Let $d=2, k>0$, and $0<\varepsilon<1$. Assume that the system is nonresonant. Then for all $K \Subset \mathbb{R}^{2} \backslash \bar{B}_{1}$,

$$
\left\|u_{c}-u \circ F_{0}^{-1}\right\|_{H^{1}(K)} \leq \frac{C}{|\ln \varepsilon|}\|f\|_{L^{2}}
$$

\footnotetext{
${ }^{4}$ The existence of $v$ is a consequence of the Fredholm alternative theory (see also part (ii) of Lemma 3.3).
} 
for some positive constant $C$ depending on $K, k, a$, and $\sigma$, but independent of $\varepsilon$ and $f$. We also have that $u_{c} \rightarrow C l_{2}(f)$ weakly in $H^{1}\left(B_{1}\right)$. As a consequence,

$$
\lim _{\varepsilon \rightarrow 0}\left\|u_{c}-C l_{2}(f)\right\|_{L^{2}\left(B_{1}\right)}=0 .
$$

Remark 1.9. From Theorem 1.8, the limit of the field in $D$ is 0 when the cloaked region is passive in the two-dimensional nonresonant case.

We also show in the following proposition that if $k$ is small enough (the smallness condition depends only on the bounds of $a$ and $\sigma$ ), then the system is nonresonant. Proposition 1.10 will be proved in Section 3.

Proposition 1.10. Let $0<c_{1}<c_{2}<0$. Assume that $c_{1}|\xi|^{2} \leq\langle a \xi, \xi\rangle \leq c_{2}|\xi|^{2}$, $0 \leq \Im(\sigma) \leq c_{2}, c_{1}<\Re(\sigma)<c_{2}$ in $B_{1}$. There exists $k_{0}>0$, depending only on $c_{1}$ and $c_{2}$, such that if $k<k_{0}$, then the system is nonresonant.

The following proposition, which will be proved in Section 4, establishes the results mentioned in Property (3).

Proposition 1.11. Let $d=3$ and $k>0$. Assume that $M \neq 0$ and fix an element $\mathbf{e} \in M$ such that $\|\mathbf{e}\|_{L^{2}\left(B_{1}\right)}=1$. Let $u_{c}$ be the solution of (1.4) with $f=0$ in $\mathbb{R}^{3} \backslash B_{1}$ and $f=\mathbf{e}$ in $B_{1}$.

(i) We have

$$
\liminf _{\varepsilon \rightarrow 0} \varepsilon\left\|u_{c}\right\|_{H^{1}\left(B_{1}\right)}>0 .
$$

(ii) Assume in addition that $\mathbf{e}$ is radial, and that $a$ and $\sigma$ are isotropic and homogeneous in $B_{1}$; i.e., $a=\lambda_{1} I$ and $\sigma=\lambda_{2}$ for some positive constants $\lambda_{1}$ and $\lambda_{2}$. Then

$$
\liminf _{\varepsilon \rightarrow 0}\left\|u_{c}\right\|_{L^{2}\left(B_{4} \backslash B_{2}\right)}>0 .
$$

Concerning the two-dimensional resonant case, we have the following proposition, which establishes the results in Property (5) and is proved in Section 5.

Proposition 1.12. Let $d=2$ and $k>0$. Assume that the system is resonant. Define

$$
N=\left\{\psi \in W^{1}\left(\mathbb{R}^{2}\right): \psi \text { satisfies }(1.9)\right\} .
$$

Fix an element $\mathbf{e} \in N$ such that $\|\mathbf{e}\|_{L^{2}\left(B_{1}\right)}=1$. Let $u_{c} \in H_{\mathrm{loc}}^{1}\left(\mathbb{R}^{2}\right)$ be the unique outgoing solution of (1.4) with $f=0$ in $\mathbb{R}^{2} \backslash B_{1}$ and $f=\mathbf{e}$ in $B_{1}$.

(i) We have

$$
\liminf _{\varepsilon \rightarrow 0}\left\|u_{c}\right\|_{H^{1}\left(B_{1}\right)}=+\infty .
$$

(ii) Assume in addition that $\mathbf{e}$ is radial, and that $a$ and $\sigma$ are isotropic and homogeneous in $B_{1}$; i.e., $a=\lambda_{1} I$ and $\sigma=\lambda_{2}$ for some positive constants $\lambda_{1}$ and $\lambda_{2}$. Then

$$
\liminf _{\varepsilon \rightarrow 0}\left\|u_{c}\right\|_{L^{2}\left(B_{4} \backslash B_{2}\right)}>0 .
$$


Concerning the instability of the approximate cloaking with respect to the material parameters inside the cloaked region, we establish the following result, which is proved in Section 6:

Proposition 1.13. Let $d=2,3, k>0$, and $\eta \in \mathbb{R}^{d}$ with $|\eta|=1$. There exist a positive number $\sigma_{0}>0$ and a family of positive numbers $\left(\sigma_{\varepsilon}\right)$ such that

$$
\left\{\begin{aligned}
0 & <\liminf _{\varepsilon \rightarrow 0} \varepsilon^{-1}\left|\sigma_{\varepsilon}-\sigma_{0}\right| \\
& \leq \limsup _{\varepsilon \rightarrow 0} \varepsilon^{-1}\left|\sigma_{\varepsilon}-\sigma_{0}\right|<+\infty \quad \text { if } d=3, \\
0 & <\liminf _{\varepsilon \rightarrow 0}|\ln \varepsilon|\left|\sigma_{\varepsilon}-\sigma_{0}\right| \\
& \leq \limsup _{\varepsilon \rightarrow 0}|\ln \varepsilon|\left|\sigma_{\varepsilon}-\sigma_{0}\right|<+\infty \quad \text { if } d=2,
\end{aligned}\right.
$$

and

$$
\liminf _{\varepsilon \rightarrow 0}\left\|u_{c, s}\right\|_{L^{2}\left(B_{4} \backslash B_{2}\right)}>0 .
$$

Here $u_{c, s} \in H_{\mathrm{loc}}^{1}\left(\mathbb{R}^{d}\right)$ satisfies the outgoing condition and $u_{c}(x):=u_{c, s}(x)+$ $e^{i k \eta \cdot x}$ is a solution of the equation

$$
\operatorname{div}\left(A_{c} \nabla u_{c}\right)+k^{2} \Sigma_{c} u_{c}=0 .
$$

Here $\left(A_{c}, \Sigma_{c}\right)$ is defined in (1.5) with $a=I$ and $\sigma=\sigma_{\varepsilon}$.

Remark 1.14. We recall that when the parameters $a$ and $\sigma$ are fixed and the cloaked region is passive, cloaking is achieved in the three-dimensional and two-dimensional nonresonant cases (see Theorems 1.4 and 1.8). Still, Proposition 1.13 does not contradict this fact since it holds under condition (1.11), which is invalid for fixed $a_{\varepsilon}$ and $\sigma_{\varepsilon}$.

Our approach to Theorems 1.4 and 1.8 is based on the study of the effect of a small inclusion. The study of approximate cloaking based on the effect of a small inclusion was discussed in $[11,12,16,18,19,20]$. It is well-known that when material parameters inside a small inclusion are, roughly speaking, bounded from below and above by positive constants, the effect of the small inclusion is small (see, e.g., [25]). Without this assumption, the effect of the inclusion is not small (see, e.g., $[11,18]$ ) unless there is an appropriate lossy layer (see $[11,18,20]$ ). In our setting, the boundedness assumption is violated and no lossy layer is used. Nevertheless, the effect of the small inclusion is still small (in the nonresonant case) due to the special structure induced from (1.2). The starting point of our approach relies on the following well-known fact:

Proposition 1.15. Let $d \geq 2, k>0$, A be a bounded matrix-valued function, $\Sigma$ be a bounded complex function defined on $\mathbb{R}^{d}, h \in L^{2}\left(\mathbb{R}^{d}\right), F: \mathbb{R}^{d} \rightarrow \mathbb{R}^{d}$ be Lipschitz, surjective, and invertible with $F(x)=x$ on $\mathbb{R}^{d} \backslash B_{2}$, and $c<\operatorname{det} D F<$ $1 / c$ a.e., $x \in \mathbb{R}^{d}$ for some positive constant $c$. Then $u \in H_{\mathrm{loc}}^{1}\left(\mathbb{R}^{d}\right)$ is a solution of

$$
\operatorname{div}(A \nabla u)+k^{2} \Sigma u=h \quad \text { in } \mathbb{R}^{d}
$$


if and only if $v:=u \circ F^{-1} \in H_{\mathrm{loc}}^{1}\left(\mathbb{R}^{d}\right)$ is a solution of

$$
\operatorname{div}\left(F_{*} A \nabla v\right)+k^{2} F_{*} \Sigma v=F_{*} h \quad \text { in } \mathbb{R}^{d},
$$

where $F_{*} A$ and $F_{*} \Sigma$ are defined in (1.5), and $F_{*} h$ is similarly defined as $F_{*} \Sigma$. Moreover, $u=v$ outside $B_{2}$.

Finally, we want to mention that the approximate cloaking for the wave equation has been recently studied in [21], where an appropriate lossy layer is used.

The paper is organized as follows: Section 2 is devoted to the proof of Theorem 1.4. In Section 3 we prove Theorem 1.8 and Proposition 1.10. The proofs of Propositions 1.11, 1.12, and 1.13 are presented in Sections 4, 5, and 6, respectively.

After finishing the present paper, we became aware of related work of Lassas and Zhou [13], in which they obtained a nonlocal boundary condition at the inner cloak interface for a spherical domain in the two-dimensional nonresonant case. Their definition of resonance is given in terms of constraints on Bessel functions [13, eq. (31)], and their boundary condition [13, eq. (41)] is in a different form from what we present here.

\section{Proof of Theorem 1.4}

It is clear from the definition of $\left(A_{c}, \Sigma_{c}\right)$ in (1.5) that $A_{c}=F_{\varepsilon *} A_{\varepsilon}$ and $\Sigma_{c}=$ $F_{\varepsilon *} \Sigma_{\varepsilon}$ where

$$
A_{\varepsilon}, \Sigma_{\varepsilon}= \begin{cases}I, 1 & \text { if } x \in \mathbb{R}^{3} \backslash B_{\varepsilon}, \\ \frac{1}{\varepsilon} a(x / \varepsilon), \frac{1}{\varepsilon^{3}} \sigma(x / \varepsilon) & \text { if } x \in B_{\varepsilon} .\end{cases}
$$

Applying Proposition 1.15, Theorem 1.4 is a consequence of the following:

THEOREM 2.1. Let $k>0$ and $0<\varepsilon<1$. Let $u_{\varepsilon} \in H_{\text {loc }}^{1}\left(\mathbb{R}^{3}\right)$ be the unique solution of

$$
\left\{\begin{array}{l}
\operatorname{div}\left(A_{\varepsilon} \nabla u_{\varepsilon}\right)+k^{2} \Sigma_{\varepsilon} u_{\varepsilon}=F_{\varepsilon}^{-1} * f \text { in } \mathbb{R}^{3}, \\
u_{\varepsilon} \text { satisfies the outgoing condition. }
\end{array}\right.
$$

In the resonant case, assume in addition that $\int_{B_{1}} f \overline{\mathbf{e}}=0$ for all $\mathbf{e} \in M$. Then for all $r>0$, there exists a constant $C=C(r, k, a, \sigma)$ that is independent of $\varepsilon$ and $f$ such that

$$
\left\|u_{\varepsilon}-u\right\|_{H^{1}\left(B_{3 r} \backslash B_{2 r}\right)} \leq C \varepsilon\|f\|_{L^{2}} .
$$

Moreover, if $U_{\varepsilon}(x)=u_{\varepsilon}(\varepsilon x)$, then $U_{\varepsilon} \rightarrow C l_{3}(f)$ weakly in $H^{1}\left(B_{1}\right)$. Consequently,

$$
\lim _{\varepsilon \rightarrow 0}\left\|U_{\varepsilon}-C l_{3}(f)\right\|_{L^{2}\left(B_{1}\right)}=0 .
$$

Here the operator $\mathrm{Cl}_{3}$ is given in Definition 1.5.

The rest of this section will be devoted to the proof of Theorem 2.1. 


\subsection{Preliminaries}

In this section we present some lemmas that will be used in the proof of Theorem 2.1. Instead of dealing with the unit ball $B_{1}$, we will present results for a smooth open subset $D$ of $\mathbb{R}^{3}$. We will also suppose that $D \subset B_{1}$ and $\mathbb{R}^{3} \backslash D$ is connected. We first recall the following result [18, lemma 2.2], which will be useful in our analysis.

LeMmA 2.2. Let $0<\varepsilon<1$ and $g_{\varepsilon} \in H^{1 / 2}(\partial D)$. Assume that $v_{\varepsilon} \in H_{\mathrm{loc}}^{1}\left(\mathbb{R}^{3} \backslash \bar{D}\right)$ is the unique solution of

$$
\begin{cases}\Delta v_{\varepsilon}+\varepsilon^{2} v_{\varepsilon}=0 & \text { in } \mathbb{R}^{3} \backslash \bar{D}, \\ v_{\varepsilon}=g_{\varepsilon} & \text { on } \partial D, \\ v_{\varepsilon} \text { satisfies the outgoing condition. } & \end{cases}
$$

(i) We have

$$
\left\|v_{\varepsilon}\right\|_{H^{1}\left(B_{r} \backslash \bar{D}\right)} \leq C_{r}\left\|g_{\varepsilon}\right\|_{H^{1 / 2}(\partial D)} \quad \forall r>5
$$

and

$$
\varepsilon^{1 / 2}\left\|v_{\varepsilon}\right\|_{L^{2}\left(B_{4 r / \varepsilon} \backslash B_{r / \varepsilon}\right)} \leq C_{r}\left\|g_{\varepsilon}\right\|_{H^{1 / 2}(\partial D)}
$$

for some positive constants $C_{r}=C(r, D)$.

(ii) Assume in addition that $g_{\varepsilon} \rightarrow g$ weakly in $H^{1 / 2}(\partial D)$ as $\varepsilon \rightarrow 0$. Then $v_{\varepsilon} \rightarrow v$ weakly in $H_{\text {loc }}^{1}\left(\mathbb{R}^{3} \backslash \bar{D}\right)$ where $v \in W^{1}\left(\mathbb{R}^{3} \backslash \bar{D}\right)$ (the space $W^{1}$ is defined in (1.7)) is the unique solution of

$$
\begin{cases}\Delta v=0 & \text { in } \mathbb{R}^{3} \backslash \bar{D}, \\ v=g & \text { on } \partial D .\end{cases}
$$

Proof. Inequalities (2.2), and (2.3) with $r=1$ are in [18, lemma 2.2]. ${ }^{5}$ They are consequences of the fact that the fundamental solution of the Helmholtz equation converges to the fundamental solution of the Laplace equation as the frequency goes to 0 in three dimensions. The proof of (2.3) in the general case follows in the same manner. Part (ii) follows from part (i) as follows: From part (i), one may assume that $v_{\varepsilon} \rightarrow v$ weakly in $H_{\text {loc }}^{1}\left(\mathbb{R}^{3} \backslash D\right.$ ) (up to a subsequence) and $v$ satisfies (2.4). Using the representation formula and the fact that the fundamental solution of the Helmholtz equation converges to the fundamental solution of the Laplace equation as the frequency goes to 0 in three dimensions, one can prove that $v \in W^{1}\left(\mathbb{R}^{3} \backslash \bar{D}\right)$. Since (2.4) has a unique solution $v \in W^{1}\left(\mathbb{R}^{3} \backslash \bar{D}\right)$ (see, e.g., $[17$, theorem 2.5 .14 , p. 64$])$, the result holds for the whole family $\left(v_{\varepsilon}\right)$. The details of the proof are left to the reader.

In what follows, $a$ denotes a real symmetric matrix-valued function and $\sigma$ denotes a complex function defined in $D$. We also assume that $a$ is uniformly elliptic and $\sigma$ satisfies $0<\operatorname{essinf} \Re \sigma \leq \operatorname{ess} \sup \Re \sigma<+\infty$ and $0 \leq \operatorname{essinf} \Im \sigma \leq$

\footnotetext{
${ }^{5}$ There is a typo in [18, eq. (2.4)] for $d=3$, where the term $\varepsilon^{1 / 2}$ is missing.
} 
ess $\sup \Im \sigma<+\infty$. We define

$$
\begin{gathered}
M:=\left\{\psi \in H^{1}(D): \operatorname{div}(a \nabla \psi)+k^{2} \sigma \psi=0 \text { in } D\right. \\
\text { and } a \nabla \psi \cdot \eta=0 \text { on } \partial D\} .
\end{gathered}
$$

The following lemma establishes the uniqueness of $(v, w)$ in Definition 1.5. This lemma is also used in the proof of Lemma 2.4.

Lemma 2.3. Assume that the system is resonant, i.e., $M \neq\{0\}$. Then there exists no nonzero solution $(v, w)$ in $W^{1}\left(\mathbb{R}^{3}\right) \times M^{\perp}$ of the systems

$$
\begin{aligned}
& \begin{cases}\Delta v=0 & \text { in } \mathbb{R}^{3} \backslash \bar{D}, \\
\operatorname{div}(a \nabla v)+k^{2} \sigma v=0 & \text { in } D, \\
\operatorname{div}(a \nabla w)+k^{2} \sigma w=0 & \text { in } D,\end{cases} \\
& \begin{cases}\left.a \nabla v \cdot \eta\right|_{\text {int }}=0 & \text { on } \partial D, \\
\left.\frac{\partial v}{\partial \eta}\right|_{\text {ext }}-a \nabla w \cdot \eta=0 & \text { on } \partial D .\end{cases}
\end{aligned}
$$

Here $M$ and $M^{\perp}$ are defined in (2.5) and (1.8), respectively.

Proof. Since $v \in W^{1}\left(\mathbb{R}^{3} \backslash \bar{D}\right)$, it follows from (2.6) that

$$
\int_{\mathbb{R}^{3} \backslash D}|\nabla v|^{2}=-\left.\int_{\partial D} \frac{\partial v}{\partial \eta}\right|_{\mathrm{ext}} \bar{v}=-\int_{\partial D}(a \nabla w \cdot \eta) \bar{v} .
$$

On the other hand, from (2.6), we have

$$
\int_{D}\langle a \nabla v, \nabla v\rangle-\int_{D} k^{2} \sigma|v|^{2}=0
$$

This implies that $v=0$ on the set $\{\Im \sigma>0\}$. Thus we deduce from (2.6) that

$$
\int_{\partial D}(a \nabla w \cdot \eta) \bar{v}=\int_{D} a \nabla w \nabla \bar{v}-\int_{D} k^{2} \sigma w \bar{v}=\overline{\int_{D} a \nabla v \nabla \bar{w}-\int_{D} k^{2} \sigma v \bar{w}}
$$

and

$$
\int_{D} a \nabla v \nabla \bar{w}-\int_{D} k^{2} \sigma v \bar{w}=\int_{\partial D}\left(\left.a \nabla v \cdot \eta\right|_{\text {int }}\right) \bar{w}=0 .
$$

A combination of (2.7), (2.8), and (2.9) yields $v=0$ in $\mathbb{R}^{3} \backslash D$. It follows that, by (2.6) and the unique continuation principle,

$$
v=0 \quad \text { in } \mathbb{R}^{3} .
$$

From (2.6), $a \nabla w \cdot \eta=0$. Hence $w=0$ since $w \in M^{\perp}$ and $\operatorname{div}(a \nabla w)+k^{2} \sigma w=$ 0.

We now establish the crucial ingredient in the proof of Theorem 2.1. 
LeMMA 2.4. Let $0<\varepsilon<1$ and $k>0$. Let $\theta_{\varepsilon} \in L^{2}(D), g_{\varepsilon} \in H^{-1 / 2}(\partial D)$, and $v_{\varepsilon} \in H_{\mathrm{loc}}^{1}\left(\mathbb{R}^{3}\right)$ be the unique solution of the system

$$
\begin{cases}\Delta v_{\varepsilon}+\varepsilon^{2} k^{2} v_{\varepsilon}=0 & \text { in } \mathbb{R}^{3} \backslash \bar{D}, \\ \operatorname{div}\left(a \nabla v_{\varepsilon}\right)+k^{2} \sigma v_{\varepsilon}=\theta_{\varepsilon} & \text { in } D, \\ \left.\frac{\partial v_{\varepsilon}}{\partial \eta}\right|_{\text {ext }}-\left.\frac{1}{\varepsilon} a \nabla v_{\varepsilon} \cdot \eta\right|_{\text {int }}=g_{\varepsilon} & \text { on } \partial D, \\ v_{\varepsilon} \text { satisfies the outgoing condition. } & \end{cases}
$$

In the case $M \neq\{0\}$ ( $M$ is defined in (2.5)), assume in addition that $\int_{B_{1}} \theta_{\varepsilon} \overline{\mathbf{e}}=0$ for all $\mathbf{e} \in M$. We have

$$
\left\|v_{\varepsilon}\right\|_{H^{1}\left(B_{5}\right)} \leq C\left(\left\|\theta_{\varepsilon}\right\|_{L^{2}(D)}+\left\|g_{\varepsilon}\right\|_{H^{-1 / 2}(\partial D)}\right)
$$

for some positive constant $C$ depending only on $k, a, \sigma$, and $D$ but independent of $\varepsilon, \theta_{\varepsilon}$, and $g_{\varepsilon}$. Assume that $\theta_{\varepsilon} \rightarrow \theta$ weakly in $L^{2}(D)$ and $g_{\varepsilon} \rightarrow g$ weakly in $H^{-1 / 2}(\partial D)$ as $\varepsilon \rightarrow 0$. Then $v_{\varepsilon} \rightarrow v$ weakly in $H^{1}(D)$. Consequently,

$$
\lim _{\varepsilon \rightarrow 0}\left\|v_{\varepsilon}-v\right\|_{L^{2}(D)}=0 \text {. }
$$

Here

(i) in the nonresonant case, $M=\{0\}, v \in H^{1}(D)$ is the unique solution of the system

$$
\begin{cases}\operatorname{div}(a \nabla v)+k^{2} \sigma v=\theta & \text { in } D, \\ a \nabla v \cdot \eta=0 & \text { on } \partial D ;\end{cases}
$$

(ii) in the resonant case, $M \neq\{0\}$, $v$ is the first component of the pair $(v, w) \in$ $W^{1}\left(\mathbb{R}^{3}\right) \times M^{\perp}$ that is the unique solution of the systems

$$
\begin{aligned}
& \begin{cases}\Delta v=0 & \text { in } \mathbb{R}^{3} \backslash \bar{D}, \\
\operatorname{div}(a \nabla v)+k^{2} \sigma v=\theta & \text { in } D, \\
\operatorname{div}(a \nabla w)+k^{2} \sigma w=0 & \text { in } D,\end{cases} \\
& \begin{cases}\left.a \nabla v \cdot \eta\right|_{\text {int }}=0 & \text { on } \partial D, \\
\left.\frac{\partial v}{\partial \eta}\right|_{\mathrm{ext}}-a \nabla w \cdot \eta=g & \text { on } \partial D .\end{cases}
\end{aligned}
$$

Here $M^{\perp}$ is defined by (1.8).

Remark 2.5. The uniqueness of $(v, w)$ follows from Lemma 2.3. The existence of $(v, w)$ will be proved in the proof of Lemma 2.4.

PROOF. We first prove that

$$
\left\|v_{\varepsilon}\right\|_{L^{2}\left(B_{5}\right)} \leq C\left(\left\|\theta_{\varepsilon}\right\|_{L^{2}(D)}+\left\|g_{\varepsilon}\right\|_{H^{-1 / 2}(\partial D)}\right)
$$

by contradiction. Suppose that there exist $\left(\theta_{n}\right) \subset L^{2}(D)$ (we also assume that $\int_{D} \theta_{n} \overline{\mathbf{e}}=0$ for any $\mathbf{e} \in M$ in the resonant case $),\left(g_{n}\right) \subset H^{-1 / 2}(\partial D)$, and $\varepsilon_{n} \rightarrow 0$ 
such that

$$
\left\|v_{n}\right\|_{L^{2}\left(B_{5}\right)}=1 \quad \text { and } \quad \lim _{n \rightarrow \infty}\left(\left\|\theta_{n}\right\|_{L^{2}(D)}+\left\|g_{n}\right\|_{H^{-1 / 2}(\partial D)}\right)=0 .
$$

Here $v_{n} \in H_{\mathrm{loc}}^{1}\left(\mathbb{R}^{3}\right)$ is the unique solution of

$$
\begin{cases}\Delta v_{n}+\varepsilon_{n}^{2} k^{2} v_{n}=0 & \text { in } \mathbb{R}^{3} \backslash \bar{D}, \\ \operatorname{div}\left(a \nabla v_{n}\right)+k^{2} \sigma v_{n}=\theta_{n} & \text { in } D, \\ \left.\frac{\partial v_{n}}{\partial \eta}\right|_{\text {ext }}-\left.\frac{1}{\varepsilon_{n}} a \nabla v_{n} \cdot \eta\right|_{\text {int }}=g_{n} & \text { on } \partial D, \\ v_{n} \text { satisfies the outgoing condition. } & \end{cases}
$$

Multiplying system (2.15) by $\bar{v}_{n}$ (the conjugate of $v_{n}$ ) and integrating the expression obtained over $B_{4}$, we have

$$
\begin{aligned}
& \int_{B_{4} \backslash D}\left|\nabla v_{n}\right|^{2}-\varepsilon_{n}^{2} k^{2} \int_{B_{4} \backslash D}\left|v_{n}\right|^{2} \\
&+\frac{1}{\varepsilon_{n}} \int_{D}\left\langle a \nabla v_{n}, \nabla v_{n}\right\rangle-\frac{1}{\varepsilon_{n}} \int_{D} k^{2} \sigma\left|v_{n}\right|^{2} \\
&=\int_{\partial B_{4}} \frac{\partial v_{n}}{\partial r} \bar{v}_{n}-\int_{\partial D} g_{n} \bar{v}_{n}-\frac{1}{\varepsilon_{n}} \int_{D} \theta_{n} \bar{v}_{n} .
\end{aligned}
$$

Applying Lemma 2.2, we deduce from (2.14) and (2.16) that

$$
\int_{D}\left|\nabla v_{n}\right|^{2} \leq C
$$

which implies, since $\left\|v_{n}\right\|_{L^{2}(D)} \leq 1$,

$$
\left\|v_{n}\right\|_{H^{1 / 2}(\partial D)} \leq C .
$$

Using Lemma 2.2, from (2.14), (2.15), and (2.17), we have

$$
\left\|v_{n}\right\|_{H^{1}\left(B_{r}\right)} \leq C_{r} \quad \forall r>0 .
$$

Case 1. The nonresonant case: $M=\{0\}$. From (2.18) and Lemma 2.2(ii), one may assume that $v_{n} \rightarrow v$ weakly in $H_{\text {loc }}^{1}\left(\mathbb{R}^{3}\right)$ and $v_{n} \rightarrow v$ in $L^{2}\left(B_{5}\right)$ for some $v \in W^{1}\left(\mathbb{R}^{3}\right)$ such that

$$
\begin{cases}\Delta v=0 & \text { in } \mathbb{R}^{3} \backslash \bar{D}, \\ \operatorname{div}(a \nabla v)+k^{2} \sigma v=0 & \text { in } D, \\ \left.a \nabla v \cdot \eta\right|_{\text {int }}=0 & \text { on } \partial D .\end{cases}
$$

Since $M=\{0\}$, it follows that

$$
v=0 \quad \text { in } D \text {. }
$$


Hence

$$
\begin{cases}\Delta v=0 & \text { in } \mathbb{R}^{3} \backslash \bar{D}, \\ v=0 & \text { on } \partial D\end{cases}
$$

Since $v \in W^{1}\left(\mathbb{R}^{3} \backslash \bar{D}\right.$ ) (by Lemma 2.2), it follows that (see, e.g., [17, theorem 2.5.14, p. 64])

$$
v=0 \quad \text { in } \mathbb{R}^{3} \backslash D .
$$

Combining (2.19) and (2.20) yields $v=0$. We have a contradiction since

$$
\|v\|_{L^{2}\left(B_{5}\right)}=\lim _{n \rightarrow \infty}\left\|v_{n}\right\|_{L^{2}\left(B_{5}\right)}=1 .
$$

Case 2. The resonant case: $M \neq\{0\}$.

Since $\int_{D} \theta_{n} \overline{\mathbf{e}}=0$ for any $\mathbf{e} \in M$, it follows from the Fredholm alternative theory (see, e.g., $[1,14]$ ) that there exists a unique solution $v_{1, n} \in M^{\perp}$ of the system

$$
\begin{cases}\operatorname{div}\left(a \nabla v_{1, n}\right)+k^{2} \sigma v_{1, n}=\theta_{n} & \text { in } D \\ a \nabla v_{1, n} \cdot \eta=0 & \text { on } \partial D\end{cases}
$$

Moreover, we have

$$
\left\|v_{1, n}\right\|_{H^{1}(D)} \leq C\left\|\theta_{n}\right\|_{L^{2}(D)}
$$

for some positive constant $C$ independent of $\theta_{n}$. Let $v_{2, n}$ be the projection of $v_{n}-v_{1, n}$ into $M$, i.e., $v_{2, n} \in M$ and $v_{n}-v_{1, n}-v_{2, n} \in M^{\perp}$. Set

$$
w_{n}=\frac{1}{\varepsilon_{n}}\left(v_{n}-v_{1, n}-v_{2, n}\right) \quad\left(\in M^{\perp}\right) \text {. }
$$

Then

$$
\left.a \nabla v_{n} \cdot \eta\right|_{\text {int }}=\varepsilon_{n} a \nabla w_{n} \cdot \eta \quad \text { on } \partial D .
$$

We deduce from (2.15) and the definition of $M$ that

$$
\begin{cases}\operatorname{div}\left(a \nabla w_{n}\right)+k^{2} \sigma w_{n}=0 & \text { in } D, \\ a \nabla w_{n} \cdot \eta=\left.\frac{\partial v_{n}}{\partial \eta}\right|_{\mathrm{ext}}-g_{n} & \text { on } \partial D .\end{cases}
$$

Combining (2.14), (2.18), and (2.25) yields

$$
\left\|a \nabla w_{n} \cdot \eta\right\|_{H^{-1 / 2}(\partial D)} \leq\left\|\left.\frac{\partial v_{n}}{\partial \eta}\right|_{\mathrm{ext}}\right\|_{H^{-1 / 2}(\partial D)}+\left\|g_{n}\right\|_{H^{-1 / 2}(\partial D)} \leq C .
$$

Since $\operatorname{div}\left(a \nabla w_{n}\right)+k^{2} \sigma w_{n}=0$ in $D$ and $w_{n} \in M^{\perp}$, it follows that

$$
\left\|w_{n}\right\|_{H^{1}(D)} \leq C \text {. }
$$

From (2.18), (2.26), and the fact that $w_{n} \in M^{\perp}$, one may assume that

$$
\begin{cases}v_{n} \rightarrow v & \text { weakly in } H_{\mathrm{loc}}^{1}\left(\mathbb{R}^{3}\right), \\ w_{n} \rightarrow w \in M^{\perp} & \text { weakly in } H^{1}(D) .\end{cases}
$$


By Lemma 2.2, it follows from (2.14), (2.15), (2.24), and (2.25) that $(v, w) \in$ $W^{1}\left(\mathbb{R}^{3}\right) \times M^{\perp}$ is a solution of the system

$$
\left\{\begin{array} { l l } 
{ \Delta v = 0 } & { \text { in } \mathbb { R } ^ { 3 } \backslash \overline { D } , } \\
{ \operatorname { d i v } ( a \nabla v ) + k ^ { 2 } \sigma v = 0 } & { \text { in } D , } \\
{ \operatorname { d i v } ( a \nabla w ) + k ^ { 2 } \sigma w = 0 } & { \text { in } D , }
\end{array} \quad \text { and } \quad \left\{\begin{array}{ll}
\left.a \nabla v \cdot \eta\right|_{\text {int }}=0 & \text { on } \partial D \\
\left.\frac{\partial v}{\partial \eta}\right|_{\text {ext }}=a \nabla w \cdot \eta & \text { on } \partial D .
\end{array}\right.\right.
$$

From Lemma 2.3 we have that $v=0$ and thus a contradiction since $\|v\|_{L^{2}\left(B_{5}\right)}=1$.

From Cases 1 and 2, (2.13) is proved. Hence we obtain (2.10) by using (2.13) and applying the same arguments used to get (2.18). The conclusion of the second part is a consequence of (2.10) and can be processed as follows: From (2.10), one may assume that $v_{\varepsilon} \rightarrow v$ weakly in $H^{1}(D)$ (up to a subsequence). In the nonresonant case, $v \in H^{1}(D)$ is a solution of the system (2.11). Since (2.11) has a unique solution $v \in H^{1}(D)$, the conclusion in this case holds for the whole family. In the resonant case, applying the same decomposition as in (2.23) and using the same facts as in (2.15), (2.24), and (2.25), one may assume that (up to a subsequence)

$$
\begin{cases}v_{\varepsilon} \rightarrow v \in W^{1}\left(\mathbb{R}^{3}\right) & \text { weakly in } H_{\mathrm{loc}}^{1}\left(\mathbb{R}^{3}\right), \\ w_{\varepsilon} \rightarrow w \in M^{\perp} & \text { weakly in } H^{1}(D),\end{cases}
$$

and $(v, w) \in W^{1}\left(\mathbb{R}^{3}\right) \times M^{\perp}$ satisfies system (2.12). Since system (2.12) has a unique solution in $(v, w) \in W^{1}\left(\mathbb{R}^{3}\right) \times M^{\perp}$ (by Lemma 2.3), the conclusion holds for the whole family.

\subsection{Proof of Theorem 2.1.}

We follow the method used in the proof of [18, theorem 2.1]. Let $u_{1, \varepsilon} \in$ $H_{\text {loc }}^{1}\left(\mathbb{R}^{3}\right)$ be the unique solution of

$$
\begin{cases}\Delta u_{1, \varepsilon}+k^{2} u_{1, \varepsilon}=f\left(=F_{\varepsilon}^{-1} f\right) & \text { in } \mathbb{R}^{3} \backslash \bar{B}_{\varepsilon} \\ u_{1, \varepsilon}=0 & \text { in } B_{\varepsilon} \\ u_{1, \varepsilon} \text { satisfies the outgoing condition. } & \end{cases}
$$

Define

$$
w_{1, \varepsilon}=u_{1, \varepsilon}-u \quad \text { and } \quad w_{2, \varepsilon}=u_{\varepsilon}-u_{1, \varepsilon} .
$$

We claim that

$$
\left\|w_{1, \varepsilon}\right\|_{H^{1}\left(B_{4 r} \backslash B_{r}\right)} \leq C_{r} \varepsilon\|f\|_{L^{2}}
$$

and

$$
\left\|w_{2, \varepsilon}\right\|_{H^{1}\left(B_{4 r} \backslash B_{r}\right)} \leq C_{r} \varepsilon\|f\|_{L^{2}} .
$$


Proof OF Claim (2.27). From the definition of $w_{1, \varepsilon}$, it follows that $w_{1, \varepsilon} \in$ $H_{\mathrm{loc}}^{1}\left(\mathbb{R}^{3}\right)$ and $w_{1, \varepsilon}$ satisfies

$$
\begin{cases}\Delta w_{1, \varepsilon}+k^{2} w_{1, \varepsilon}=0 & \text { in } \mathbb{R}^{3} \backslash \bar{B}_{\varepsilon}, \\ w_{1, \varepsilon}=-u & \text { in } \partial B_{\varepsilon}, \\ w_{1, \varepsilon} \text { satisfies the outgoing condition. } & \end{cases}
$$

Define $W_{1, \varepsilon}(x)=w_{1, \varepsilon}(\varepsilon x)$. Then $W_{1, \varepsilon} \in H_{\text {loc }}^{1}\left(\mathbb{R}^{3}\right)$ and $W_{1, \varepsilon}$ satisfies

$$
\begin{cases}\Delta W_{1, \varepsilon}+\varepsilon^{2} k^{2} W_{1, \varepsilon}=0 & \text { in } \mathbb{R}^{3} \backslash \bar{B}_{1}, \\ W_{1, \varepsilon}=-u(\varepsilon \cdot) & \text { in } \partial B_{1}, \\ W_{1, \varepsilon} \text { satisfies the outgoing condition. } & \end{cases}
$$

Since $\|u(\varepsilon \cdot)\|_{H^{1 / 2}\left(\partial B_{1}\right)} \leq\|f\|_{L^{2}}$, by Lemma 2.2 , we have

$$
\varepsilon \int_{B_{4 r / \varepsilon} \backslash B_{r / \varepsilon}}\left|W_{1, \varepsilon}\right|^{2} \leq C_{r}\|f\|_{L^{2}}^{2}
$$

By a change of variables,

$$
\int_{B_{4 r} \backslash B_{r}}\left|w_{1, \varepsilon}\right|^{2} \leq C_{r} \varepsilon^{2}\|f\|_{L^{2}}^{2} .
$$

Since $\Delta w_{1, \varepsilon}+k^{2} w_{1, \varepsilon}=0$ in $\mathbb{R}^{3} \backslash \bar{B}_{\varepsilon}$, by the regularity theory of elliptic equations, Claim (2.27) follows from (2.31).

Proof OF ClAim (2.28). It is clear that $w_{2, \varepsilon} \in H_{\mathrm{loc}}^{1}\left(\mathbb{R}^{3}\right)$ is the unique solution of

$$
\begin{cases}\Delta w_{2, \varepsilon}+k^{2} w_{2, \varepsilon}=0 & \text { in } \mathbb{R}^{3} \backslash \bar{B}_{\varepsilon}, \\ \operatorname{div}\left(A_{\varepsilon} \nabla w_{2, \varepsilon}\right)+k^{2} \Sigma_{\varepsilon} w_{2, \varepsilon}=F_{\varepsilon}^{-1} * f & \text { in } B_{\varepsilon}, \\ \left.\frac{\partial w_{2, \varepsilon}}{\partial \eta}\right|_{\text {ext }}-\left.A_{\varepsilon} \nabla w_{2, \varepsilon} \cdot \eta\right|_{\text {int }}=-\frac{\partial u_{1, \varepsilon}}{\partial \eta} & \text { on } \partial B_{\varepsilon}, \\ w_{2, \varepsilon} \text { satisfies the outgoing condition. } & \end{cases}
$$

Define $W_{2, \varepsilon}(x)=w_{2, \varepsilon}(\varepsilon x)$. Then $W_{2, \varepsilon} \in H_{\mathrm{loc}}^{1}\left(\mathbb{R}^{3}\right)$ and $W_{2, \varepsilon}$ is the unique solution of

$$
\begin{cases}\Delta W_{2, \varepsilon}+\varepsilon^{2} k^{2} W_{2, \varepsilon}=0 & \text { in } \mathbb{R}^{3} \backslash \bar{B}_{1}, \\ \operatorname{div}\left(a \nabla W_{2, \varepsilon}\right)+k^{2} \sigma W_{2, \varepsilon}=f & \text { in } B_{1}, \\ \left.\frac{\partial W_{2, \varepsilon}}{\partial \eta}\right|_{\text {ext }}-\left.\frac{1}{\varepsilon} a \nabla W_{2, \varepsilon} \cdot \eta\right|_{\text {int }}=-\varepsilon \frac{\partial u_{1, \varepsilon}}{\partial \eta}(\varepsilon x) & \text { on } \partial B_{1}, \\ W_{2, \varepsilon} \text { satisfies the outgoing condition. } & \end{cases}
$$

Since $u_{1, \varepsilon}=w_{1, \varepsilon}+u$ and $W_{1, \varepsilon}=w_{1, \varepsilon}(\varepsilon \cdot)$, it follows that

$$
\varepsilon \frac{\partial u_{1, \varepsilon}}{\partial \eta}(\varepsilon x)=\frac{\partial W_{1, \varepsilon}}{\partial \eta}(x)+\varepsilon \frac{\partial u}{\partial \eta}(\varepsilon x) \quad \text { on } \partial B_{1} .
$$


From (2.30) and Lemma 2.2, we obtain

$$
\left\|\varepsilon \frac{\partial u_{1, \varepsilon}}{\partial \eta}(\varepsilon x)\right\|_{H^{-1 / 2}\left(\partial B_{1}\right)} \leq C\|f\|_{L^{2}}
$$

We deduce from Lemma 2.4 that

$$
\left\|W_{2, \varepsilon}\right\|_{H^{1}\left(B_{5}\right)} \leq C\|f\|_{L^{2}} \text {. }
$$

Applying Lemma 2.2, we have

$$
\varepsilon \int_{B_{4 r / \varepsilon} \backslash B_{r / \varepsilon}}\left|W_{2, \varepsilon}\right|^{2} \leq C_{r}\|f\|_{L^{2}}^{2} .
$$

By a change of variables,

$$
\int_{B_{4} \backslash B_{r}}\left|w_{2, \varepsilon}\right|^{2} \leq C_{r} \varepsilon^{2}\|f\|_{L^{2}}^{2} .
$$

Claim (2.28) now follows from the regularity theory of elliptic equations and the fact that $\Delta w_{2, \varepsilon}+k^{2} w_{2, \varepsilon}=0$ in $\mathbb{R}^{3} \backslash \bar{B}_{\varepsilon}$.

Thus Claims (2.27) and (2.28) are proved. Since $u_{\varepsilon}-u=w_{1, \varepsilon}+w_{2, \varepsilon},(2.1)$ follows.

The rest of the proof goes as follows. Since $u(\varepsilon \cdot) \rightarrow u(0)$, it follows from (2.30) and Lemma 2.2 that $W_{1, \varepsilon} \rightarrow W_{1}$ weakly in $H_{\text {loc }}^{1}\left(\mathbb{R}^{3} \backslash \bar{B}_{1}\right)$ where $W_{1} \in$ $W^{1}\left(\mathbb{R}^{3} \backslash \bar{B}_{1}\right)$ is the unique solution of

$$
\begin{cases}\Delta W_{1}=0 & \text { in } \mathbb{R}^{3} \backslash \bar{B}_{1}, \\ W_{1}=-u(0) & \text { in } \partial B_{1} .\end{cases}
$$

Case 1. The nonresonant case: $M=\{0\}$. By Lemma $2.4, W_{2, \varepsilon} \rightarrow \widehat{v}$ weakly in $H^{1}(B)$ where $\widehat{v}$ is the unique solution of the system

$$
\begin{cases}\operatorname{div}(a \nabla \hat{v})+k^{2} \sigma \hat{v}=f & \text { in } B_{1}, \\ \left.a \nabla \hat{v} \cdot \eta\right|_{\text {int }}=0 & \text { on } \partial B_{1} .\end{cases}
$$

Therefore, the conclusion follows in this case.

Case 2. The resonant case: $M \neq\{0\}$. By Lemma 2.4, it follows from (2.32) that $W_{2, \varepsilon} \rightarrow \hat{v}$ weakly in $H_{\text {loc }}^{1}\left(\mathbb{R}^{3}\right)$ where $\hat{v}$ is the first component of the pair $(\hat{v}, \widehat{w}) \in W^{1}\left(\mathbb{R}^{3}\right) \times M^{\perp}$, which is the unique solution of the systems

$$
\begin{aligned}
& \begin{cases}\Delta \hat{v}=0 & \text { in } \mathbb{R}^{3} \backslash \bar{B}_{1}, \\
\operatorname{div}(a \nabla \hat{v})+k^{2} \sigma \widehat{v}=f & \text { in } B_{1}, \\
\operatorname{div}(a \nabla \hat{w})+k^{2} \sigma \hat{w}=0 & \text { in } B_{1},\end{cases} \\
& \begin{cases}\left.a \nabla \hat{v} \cdot \eta\right|_{\text {int }}=0 & \text { on } \partial B_{1}, \\
\left.\frac{\partial \hat{v}}{\partial \eta}\right|_{\text {ext }}+\frac{\partial W_{1}}{\partial \eta}=a \nabla \hat{w} \cdot \eta & \text { on } \partial B_{1} .\end{cases}
\end{aligned}
$$


Hence $\widehat{v}$ depends on $u(0)$ through $W_{1}$ (see the transmission condition in (2.34) and the system of $W_{1}$ in (2.33)). Define $v_{\text {ext }}=\hat{v}+W_{1}$ for $x \in \mathbb{R}^{3} \backslash \bar{B}_{1}, v_{\text {int }}=\hat{v}$ if $x \in B_{1}$, and $w=\widehat{w}$ if $x \in B_{1}$. It follows from (2.33) and (2.34) that the triple $\left(v_{\text {ext }}, v_{\text {int }}, w\right) \in W^{1}\left(\mathbb{R}^{3} \backslash \bar{B}_{1}\right) \times H^{1}\left(B_{1}\right) \times M^{\perp}$ is the unique solution of the systems

$$
\begin{aligned}
& \begin{cases}\Delta v_{\mathrm{ext}}=0 & \text { in } \mathbb{R}^{3} \backslash \bar{B}_{1}, \\
\operatorname{div}\left(a \nabla v_{\mathrm{int}}\right)+k^{2} \sigma v_{\mathrm{int}}=f & \text { in } B_{1}, \\
\operatorname{div}(a \nabla w)+k^{2} \sigma w=0 & \text { in } B_{1},\end{cases} \\
& \begin{cases}v_{\mathrm{ext}}-v_{\text {int }}=-u(0) & \text { on } \partial B_{1}, \\
a \nabla v_{\text {int }} \cdot \eta=0 & \text { on } \partial B_{1}, \\
\frac{\partial v_{\mathrm{ext}}}{\partial \eta}=a \nabla w \cdot \eta & \text { on } \partial B_{1} .\end{cases}
\end{aligned}
$$

Since $U_{\varepsilon}=W_{2, \varepsilon}$ in $B_{1}$ and $W_{2, \varepsilon} \rightarrow \hat{v}=v_{\text {int }}=C l_{3}(f)$ in $H^{1}\left(B_{1}\right)$, we obtain the conclusion in this case.

\section{Proofs of Theorem 1.8 and Proposition 1.10}

According to Proposition 1.15, Theorem 1.8 is a consequence of the following:

Theorem 3.1. Let $d=2, k>0$, and $0<\varepsilon<1$. Define

$$
A_{\varepsilon}, \Sigma_{\varepsilon}= \begin{cases}I, 1 & \text { if } x \in \mathbb{R}^{2} \backslash B_{\varepsilon}, \\ a(x / \varepsilon), \frac{1}{\varepsilon^{2}} \sigma(x / \varepsilon) & \text { if } x \in B_{\varepsilon}\end{cases}
$$

Let $u_{\varepsilon} \in H_{\mathrm{loc}}^{1}\left(\mathbb{R}^{2}\right)$ be the unique solution of

$$
\left\{\begin{array}{l}
\operatorname{div}\left(A_{\varepsilon} \nabla u_{\varepsilon}\right)+k^{2} \Sigma_{\varepsilon} u_{\varepsilon}=F_{\varepsilon}^{-1} * f \quad \text { in } \mathbb{R}^{2}, \\
u_{\varepsilon} \text { satisfies the outgoing condition. }
\end{array}\right.
$$

Assume the system is nonresonant (the nonresonant system is defined in Definition 1.7). We have

$$
\left\|u_{\varepsilon}-u\right\|_{H^{1}\left(B_{4 r} \backslash B_{r}\right)} \leq \frac{C}{|\ln \varepsilon|}\|f\|_{L^{2}}
$$

for some $C=C(r, k, a, \sigma)$. Moreover, if $U_{\varepsilon}(x)=u_{\varepsilon}(\varepsilon x)$, then $U_{\varepsilon} \rightarrow C l_{2}(f)$ weakly in $H^{1}\left(B_{1}\right)$. Consequently,

$$
\lim _{\varepsilon \rightarrow 0}\left\|U_{\varepsilon}-C l_{2}(f)\right\|_{L^{2}\left(B_{1}\right)}=0 .
$$

In the rest of this section, we present the proofs of Theorem 3.1 and Proposition 1.10. 


\subsection{Preliminaries}

In this section we present some lemmas that will be used in the proof of Theorem 3.1. Instead of dealing with the unit ball $B_{1}$, we will present results for a smooth open subset $D$ of $\mathbb{R}^{2}$. We will also assume that $D \subset B_{1}$ and $\mathbb{R}^{2} \backslash D$ is connected. We first recall the following result [18, lemma 2.2], which will be useful in our analysis.

LEMMA 3.2. Let $0<\varepsilon<1$ and $g_{\varepsilon} \in H^{1 / 2}(\partial D)$. Assume that $\mathbb{R}^{2} \backslash D$ is connected and $v_{\varepsilon} \in H_{\mathrm{loc}}^{1}\left(\mathbb{R}^{2}\right)$ is the unique solution of

$$
\begin{cases}\Delta v_{\varepsilon}+\varepsilon^{2} v_{\varepsilon}=0 & \text { in } \mathbb{R}^{2} \backslash \bar{D}, \\ v_{\varepsilon}=g_{\varepsilon} & \text { on } \partial D, \\ v_{\varepsilon} \text { satisfies the outgoing condition. } & \end{cases}
$$

(i) We have

$$
\left\|v_{\varepsilon}\right\|_{H^{1}\left(B_{r} \backslash D\right)} \leq C_{r}\left\|g_{\varepsilon}\right\|_{H^{1 / 2}(\partial D)} \quad \forall r>5
$$

and

$$
\left\|v_{\varepsilon}\right\|_{L^{2}\left(B_{4 r / \varepsilon} \backslash B_{r / \varepsilon}\right)} \leq \frac{C_{r}}{\varepsilon|\ln \varepsilon|}\left\|g_{\varepsilon}\right\|_{H^{1 / 2}(\partial D)}
$$

for some positive constants $C_{r}=C(r, D)$.

(ii) Assume that $g_{\varepsilon} \rightarrow g$ weakly in $H^{1 / 2}(\partial D)$. Then $v_{\varepsilon} \rightarrow v$ weakly in $H_{\text {loc }}^{1}\left(\mathbb{R}^{2} \backslash D\right)$, where $v \in W^{1}\left(\mathbb{R}^{2} \backslash \bar{D}\right)$ (defined in (1.10)) is the unique solution of

$$
\begin{cases}\Delta v=0 & \text { in } \mathbb{R}^{2} \backslash \bar{D}, \\ v=g & \text { on } \partial D .\end{cases}
$$

Proof. Inequalities (3.2) and (3.3) with $r=1$ are in [18, lemma 2.2]. ${ }^{6}$ The proof of (3.3) in the general case follows in the same manner. To prove part (ii) we process as follows. Since $\left(v_{\varepsilon}\right)$ is bounded in $H_{\text {loc }}^{1}\left(\mathbb{R}^{2} \backslash D\right)$, one may assume that $v_{\varepsilon} \rightarrow v$ weakly in $H_{\text {loc }}^{1}\left(\mathbb{R}^{2} \backslash D\right)$ (up to a subsequence). Then $v \in W^{1}\left(\mathbb{R}^{2} \backslash \bar{D}\right)$. This fact is not stated in [18, lemma 2.2]; however, the proof is already there (see $[18,(2.20)$ and (2.22)]). It is clear that $v$ satisfies (3.4). Since (3.4) has a unique solution $v \in W^{1}\left(\mathbb{R}^{2} \backslash \bar{D}\right)$ (see, e.g., [17, theorem 2.5.14 and remarks on p. 64]) the conclusion holds for the whole sequence. The details of the proof are left to the reader.

The following lemma plays an important role in the proof of Theorem 3.1.

LEMMA 3.3. Let $0<\varepsilon<1, k>0, \theta_{\varepsilon} \in L^{2}(D), g_{\varepsilon} \in H^{-1 / 2}(\partial D)$, a be a real symmetric matrix-valued function, and $\sigma$ be a complex function defined on D. Assume that a is uniformly elliptic, $0<\operatorname{ess} \inf \Re \sigma \leq \operatorname{ess} \sup \Re \sigma<+\infty$,

\footnotetext{
${ }^{6}$ There is a typo in $[18,(2.4)]$ for $d=2$ where the term $\varepsilon^{1 / 2}$ must be replaced by $\varepsilon$.
} 
$0 \leq \operatorname{essinf} \Im \sigma \leq \operatorname{ess} \sup \Im \sigma<+\infty$, and the system is nonresonant. ${ }^{7}$ Let $v_{\varepsilon} \in H_{\mathrm{loc}}^{1}\left(\mathbb{R}^{2}\right)$ be the unique solution of

$$
\begin{cases}\Delta v_{\varepsilon}+\varepsilon^{2} k^{2} v_{\varepsilon}=0 & \text { in } \mathbb{R}^{2} \backslash \bar{D}, \\ \operatorname{div}\left(a \nabla v_{\varepsilon}\right)+k^{2} \sigma v_{\varepsilon}=\theta_{\varepsilon} & \text { in } D, \\ \left.\frac{\partial v_{\varepsilon}}{\partial \eta}\right|_{\text {ext }}-\left.a \nabla v_{\varepsilon} \cdot \eta\right|_{\text {int }}=g_{\varepsilon} & \text { on } \partial D, \\ v_{\varepsilon} \text { satisfies the outgoing condition. } & \end{cases}
$$

(i) We have

$$
\left\|v_{\varepsilon}\right\|_{H^{1}\left(B_{5} \backslash D\right)} \leq C\left(\left\|\theta_{\varepsilon}\right\|_{L^{2}(D)}+\left\|g_{\varepsilon}\right\|_{H^{-1 / 2}(\partial D)}\right)
$$

for some positive constant $C$ depending only on $k, a, \sigma$, and $D$ but independent of $\varepsilon, \theta_{\varepsilon}$, and $g_{\varepsilon}$.

(ii) Assume in addition that $\theta_{\varepsilon} \rightarrow \theta$ weakly in $L^{2}(D)$ and $g_{\varepsilon} \rightarrow g$ weakly in $H^{-1 / 2}(\partial D)$ as $\varepsilon \rightarrow 0$. Then $v_{\varepsilon} \rightarrow v$ weakly in $H^{1}(D)$. Consequently,

$$
\lim _{\varepsilon \rightarrow 0}\left\|v_{\varepsilon}-v\right\|_{L^{2}(D)}=0 .
$$

Here $v \in W^{1}\left(\mathbb{R}^{2}\right)$ is the unique solution of the system

$$
\begin{cases}\Delta v=0 & \text { in } \mathbb{R}^{2} \backslash \bar{D}, \\ \operatorname{div}(a \nabla v)+k^{2} \sigma v=\theta & \text { in } D, \\ \left.\frac{\partial v}{\partial \eta}\right|_{\text {ext }}-\left.a \nabla v \cdot \eta\right|_{\text {int }}=g & \text { on } \partial D .\end{cases}
$$

PROOF. We first prove that

$$
\left\|v_{\varepsilon}\right\|_{L^{2}\left(B_{5}\right)} \leq C\left(\left\|\theta_{\varepsilon}\right\|_{L^{2}(D)}+\left\|g_{\varepsilon}\right\|_{H^{-1 / 2}(\partial D)}\right)
$$

by contradiction. Suppose that there exist $\left(\theta_{n}\right) \subset L^{2}(D),\left(g_{n}\right) \subset H^{-1 / 2}(\partial D)$, and $\left(\varepsilon_{n}\right)$ such that $\left(\varepsilon_{n}\right)$ converges to 0 ,

$$
\left\|v_{n}\right\|_{L^{2}\left(B_{5}\right)}=1 \quad \text { and } \quad \lim _{n \rightarrow \infty}\left(\left\|\theta_{n}\right\|_{L^{2}(D)}+\left\|g_{n}\right\|_{H^{-1 / 2}(\partial D)}\right)=0 .
$$

Here $v_{n} \in H_{\text {loc }}^{1}\left(\mathbb{R}^{2}\right)$ is the unique solution of the system

$$
\begin{cases}\Delta v_{n}+\varepsilon_{n}^{2} k^{2} v_{n}=0 & \text { in } \mathbb{R}^{2} \backslash \bar{D}, \\ \operatorname{div}\left(a \nabla v_{n}\right)+k^{2} \sigma v_{n}=\theta_{n} & \text { in } D, \\ \left.\frac{\partial v_{n}}{\partial \eta}\right|_{\text {ext }}-\left.a \nabla v_{n} \cdot \eta\right|_{\text {int }}=g_{n} & \text { on } \partial D, \\ v_{n} \text { satisfies the outgoing condition. } & \end{cases}
$$

Applying Lemma 3.2 and the regularity theory of elliptic equations, we deduce from (3.7) that

$$
\left\|v_{n}\right\|_{H^{1}\left(B_{r} \backslash B_{3}\right)} \leq C_{r} \quad \forall r>5 .
$$

\footnotetext{
${ }^{7}$ The definition of the nonresonance in this case is the same as the case corresponding to the unit ball $B_{1}$; i.e., if $w \in W^{1}\left(\mathbb{R}^{2}\right)$ satisfies (1.9) where $B_{1}$ is replaced by $D$, then $w=0$.
} 
Multiplying system (3.8) with $\bar{v}_{n}$ (the conjugate of $v_{n}$ ) and integrating the expression obtained over $B_{4}$, we have

$$
\begin{aligned}
& \int_{B_{4} \backslash D}\left|\nabla v_{n}\right|^{2}-\varepsilon_{n}^{2} k^{2} \int_{B_{4} \backslash D}\left|v_{n}\right|^{2} \\
&+\int_{D}\left\langle a \nabla v_{n}, \nabla v_{n}\right\rangle-\int_{D} k^{2} \sigma\left|v_{n}\right|^{2} \\
&=-\int_{D} \theta_{n} \bar{v}_{n}+\int_{\partial B_{4}} \frac{\partial v_{n}}{\partial r} \bar{v}_{n}-\int_{\partial D} g_{n} \bar{v}_{n} .
\end{aligned}
$$

From (3.7) and (3.9), it follows that

$$
\int_{B_{4}}\left|\nabla v_{n}\right|^{2} \leq C
$$

Combining (3.7), (3.9), and (3.10) yields

$$
\left\|v_{n}\right\|_{H^{1}\left(B_{r}\right)} \leq C_{r} \quad \forall r>0 .
$$

Hence without loss of generality, one may assume that $v_{n} \rightarrow v$ weakly in $H_{\text {loc }}^{1}\left(\mathbb{R}^{2}\right)$ and $v_{n} \rightarrow v$ in $L_{\text {loc }}^{2}\left(\mathbb{R}^{2}\right)$, where $v \in W^{1}\left(\mathbb{R}^{2}\right)$ (by Lemma 3.2) is a solution of the system

$$
\begin{cases}\Delta v=0 & \text { in } \mathbb{R}^{2} \backslash \bar{D} \\ \operatorname{div}(a \nabla v)+k^{2} \sigma v=0 & \text { in } D \\ \left.\frac{\partial v}{\partial \eta}\right|_{\text {ext }}-\left.a \nabla v \cdot \eta\right|_{\text {int }}=0 & \text { on } \partial D .\end{cases}
$$

Since the system is nonresonant, $v=0$. We have a contradiction since

$$
\|v\|_{L^{2}\left(B_{5}\right)}=\lim _{n \rightarrow \infty}\left\|v_{n}\right\|_{L^{2}\left(B_{5}\right)}=1 .
$$

Hence (3.6) is proved. Using the same argument to obtain (3.11), we deduce part (i) of the conclusion from (3.6). Part (ii) of the conclusion follows from part (i) and the uniqueness of system (3.5).

\subsection{Proof of Theorem 3.1}

The proof of Theorem 3.1 is quite similar to the one of Theorem 2.1. However, instead of using Lemmas 2.2, 2.3, and 2.4, we apply Lemmas 3.2 and 3.3. For the convenience of the reader, we present the proof.

Let $u_{1, \varepsilon} \in H_{\text {loc }}^{1}\left(\mathbb{R}^{2}\right)$ be the unique solution of

$$
\begin{cases}\Delta u_{1, \varepsilon}+k^{2} u_{1, \varepsilon}=f\left(=F_{\varepsilon}^{-1} f\right) & \text { in } \mathbb{R}^{2} \backslash \bar{B}_{\varepsilon}, \\ u_{1, \varepsilon}=0 & \text { in } B_{\varepsilon}, \\ u_{1, \varepsilon} \text { satisfies the outgoing condition. } & \end{cases}
$$


Define

$$
w_{1, \varepsilon}=u_{1, \varepsilon}-u \quad \text { and } \quad w_{2, \varepsilon}=u_{\varepsilon}-u_{1, \varepsilon} .
$$

We claim that

$$
\left\|w_{1, \varepsilon}\right\|_{H^{1}\left(B_{4 r} \backslash B_{r}\right)} \leq \frac{C_{r}}{|\ln \varepsilon|}\|f\|_{L^{2}}
$$

and

$$
\left\|w_{2, \varepsilon}\right\|_{H^{1}\left(B_{4 r} \backslash B_{r}\right)} \leq \frac{C_{r}}{|\ln \varepsilon|}\|f\|_{L^{2}} .
$$

Proof OF Claim (3.12). From the definition of $w_{1, \varepsilon}$, it follows that $w_{1, \varepsilon} \in$ $H_{\mathrm{loc}}^{1}\left(\mathbb{R}^{2}\right)$, and $w_{1, \varepsilon}$ satisfies

$$
\begin{cases}\Delta w_{1, \varepsilon}+k^{2} w_{1, \varepsilon}=0 & \text { in } \mathbb{R}^{2} \backslash \bar{B}_{\varepsilon} \\ w_{1, \varepsilon}=-u & \text { in } \partial B_{\varepsilon} \\ w_{1, \varepsilon} \text { satisfies the outgoing condition. } & \end{cases}
$$

Define $W_{1, \varepsilon}(x)=w_{1, \varepsilon}(\varepsilon x)$. Then $W_{1, \varepsilon} \in H_{\mathrm{loc}}^{1}\left(\mathbb{R}^{2}\right)$, and $W_{1, \varepsilon}$ satisfies

$$
\begin{cases}\Delta W_{1, \varepsilon}+\varepsilon^{2} k^{2} W_{1, \varepsilon}=0 & \text { in } \mathbb{R}^{2} \backslash \bar{B}_{1}, \\ W_{1, \varepsilon}=-u(\varepsilon \cdot) & \text { in } \partial B_{1}, \\ W_{1, \varepsilon} \text { satisfies the outgoing condition. } & \end{cases}
$$

Since $\|u(\varepsilon \cdot)\|_{H^{1 / 2}\left(\partial B_{1}\right)} \leq\|f\|_{L^{2}}$, by Lemma 3.2, we have

$$
\varepsilon^{2} \int_{B_{4 r / \varepsilon} \backslash B_{r / \varepsilon}}\left|W_{1, \varepsilon}\right|^{2} \leq \frac{C_{r}}{|\ln \varepsilon|^{2}}\|f\|_{L^{2}}^{2} .
$$

By a change of variables,

$$
\int_{B_{4 r} \backslash B_{r}}\left|w_{1, \varepsilon}\right|^{2} \leq \frac{C_{r}}{|\ln \varepsilon|^{2}}\|f\|_{L^{2}}^{2} .
$$

Since $\Delta w_{1, \varepsilon}+k^{2} w_{1, \varepsilon}=0$ in $\mathbb{R}^{2} \backslash \bar{B}_{\varepsilon}$, by the regularity theory of elliptic equations, Claim (3.12) follows from (3.15).

ProOF OF CLAIM (3.13). It is clear that $w_{2, \varepsilon} \in H_{\text {loc }}^{1}\left(\mathbb{R}^{2}\right)$ is the unique solution of

$$
\begin{cases}\Delta w_{2, \varepsilon}+k^{2} w_{2, \varepsilon}=0 & \text { in } \mathbb{R}^{2} \backslash \bar{B}_{\varepsilon}, \\ \operatorname{div}\left(A_{\varepsilon} \nabla w_{2, \varepsilon}\right)+k^{2} \Sigma_{\varepsilon} w_{2, \varepsilon}=F_{\varepsilon}^{-1}{ }_{*} f & \text { in } B_{\varepsilon}, \\ \left.\frac{\partial w_{2, \varepsilon}}{\partial \eta}\right|_{\text {ext }}-\left.A_{\varepsilon} \nabla w_{2, \varepsilon} \cdot \eta\right|_{\text {int }}=-\frac{\partial u_{1, \varepsilon}}{\partial \eta} & \text { on } \partial B_{\varepsilon}, \\ w_{2, \varepsilon} \text { satisfies the outgoing condition. } & \end{cases}
$$


Define $W_{2, \varepsilon}(x)=w_{2, \varepsilon}(\varepsilon x)$. Then $W_{2, \varepsilon} \in H_{\mathrm{loc}}^{1}\left(\mathbb{R}^{2}\right)$ and $W_{2, \varepsilon}$ is the unique solution of

$$
\begin{cases}\Delta W_{2, \varepsilon}+\varepsilon^{2} k^{2} W_{2, \varepsilon}=0 & \text { in } \mathbb{R}^{2} \backslash \bar{B}_{1}, \\ \operatorname{div}\left(a \nabla W_{2, \varepsilon}\right)+k^{2} \sigma W_{2, \varepsilon}=f & \text { in } B_{1}, \\ \left.\frac{\partial W_{2, \varepsilon}}{\partial \eta}\right|_{\text {ext }}-\left.a \nabla W_{2, \varepsilon} \cdot \eta\right|_{\text {int }}=-\varepsilon \frac{\partial u_{1, \varepsilon}}{\partial \eta}(\varepsilon x) & \text { on } \partial B_{1}, \\ W_{2, \varepsilon} \text { satisfies the outgoing condition. } & \end{cases}
$$

Since $u_{1, \varepsilon}=w_{1, \varepsilon}+u$ and $W_{1, \varepsilon}=w_{1, \varepsilon}(\varepsilon \cdot)$, it follows that

$$
\varepsilon \frac{\partial u_{1, \varepsilon}}{\partial \eta}(\varepsilon x)=\frac{\partial W_{1, \varepsilon}}{\partial \eta}(x)+\varepsilon \frac{\partial u}{\partial \eta}(\varepsilon x) \quad \text { on } \partial B_{1} .
$$

From (3.14) and Lemma 3.2, we obtain

$$
\left\|\varepsilon \frac{\partial u_{1, \varepsilon}}{\partial \eta}(\varepsilon x)\right\|_{H^{-1 / 2}\left(\partial B_{1}\right)} \leq C\|f\|_{L^{2}} .
$$

We deduce from Lemma 3.3 that

$$
\left\|W_{2, \varepsilon}\right\|_{H^{1}\left(B_{5}\right)} \leq C\|f\|_{L^{2}} .
$$

Applying Lemma 3.2, we have

$$
\varepsilon^{2} \int_{B_{4 r / \varepsilon} \backslash B_{r / \varepsilon}}\left|W_{2, \varepsilon}\right|^{2} \leq \frac{C_{r}}{|\ln \varepsilon|^{2}}\|f\|_{L^{2}}^{2} .
$$

By a change of variables,

$$
\int_{B_{4} \backslash B_{r}}\left|w_{2, \varepsilon}\right|^{2} \leq \frac{C_{r}}{|\ln \varepsilon|^{2}}\|f\|_{L^{2}}^{2} .
$$

Claim (3.13) now follows from the regularity theory of elliptic equations and the fact that $\Delta w_{2, \varepsilon}+k^{2} w_{2, \varepsilon}=0$ in $\mathbb{R}^{2} \backslash \bar{B}_{\varepsilon}$.

Thus Claims (3.12) and (3.13) are proved. Since $u_{\varepsilon}-u=w_{1, \varepsilon}+w_{2, \varepsilon},(3.1)$ follows.

The rest of the proof now follows from the following facts:

(1) $W_{1, \varepsilon} \rightarrow W_{1}=-u(0)$ weakly in $H_{\text {loc }}^{1}\left(\mathbb{R}^{2} \backslash \bar{B}_{1}\right)$ since $W_{1} \in W^{1}\left(\mathbb{R}^{2} \backslash \bar{B}_{1}\right)$ is the unique solution of

$$
\begin{cases}\Delta W_{1}=0 & \text { in } \mathbb{R}^{2} \backslash \bar{B}_{1}, \\ W_{1}=-u(0) & \text { in } \partial B_{1}\end{cases}
$$

by Lemma 3.2.

(2) $W_{2, \varepsilon} \rightarrow v$ weakly in $W_{\text {loc }}^{1}\left(\mathbb{R}^{2}\right)$ where $v$ is the unique solution of the system

$$
\begin{cases}\Delta v=0 & \text { in } \mathbb{R}^{2} \backslash \bar{B}_{1}, \\ \operatorname{div}(a \nabla v)+k^{2} \sigma v=f & \text { in } B_{1}, \\ \left.\frac{\partial v}{\partial \eta}\right|_{\text {ext }}=a \nabla v \cdot \eta & \text { on } \partial B_{1}\end{cases}
$$


since $W_{1}=-u(0)$ in $\mathbb{R}^{2} \backslash B_{1}$ by statement (1), and (3.16) holds.

(3) $U_{\varepsilon}=W_{2, \varepsilon}$ in $B_{1}$ and $v=C l_{2}(f)$.

\subsection{Proof of Proposition 1.10}

Proposition 1.10 is a consequence of the following lemma:

LEMMA 3.4. Let $k>0,0<c_{1}<c_{2}<\infty, \theta \in L^{2}\left(B_{1}\right)$, and $g \in H^{1 / 2}\left(\partial B_{1}\right)$. Assume that $c_{1}|\xi|^{2} \leq\langle a \xi, \xi\rangle \leq c_{2}|\xi|^{2}, 0 \leq \Im(\sigma) \leq c_{2}$, and $c_{1}<\Re(\sigma)<c_{2}$ on $B_{1}$. Then there exists $k_{0}>0$ depending only on $c_{1}$ and $c_{2}$ such that if $0<k<k_{0}$, then there exists a unique solution $v \in W^{1}\left(\mathbb{R}^{2}\right)$ of the system

$$
\begin{cases}\Delta v=0 & \text { in } \mathbb{R}^{2} \backslash \bar{B}_{1}, \\ \operatorname{div}(a \nabla v)+k^{2} \sigma v=\theta & \text { in } B_{1}, \\ \left.\frac{\partial v}{\partial \eta}\right|_{\mathrm{ext}}=\left.a \nabla v \cdot \eta\right|_{\mathrm{int}}+g & \text { on } \partial B_{1} .\end{cases}
$$

Proof. We only prove the uniqueness of $v$. The existence of $v$ follows from the uniqueness. For this end, it suffices to prove that $v=0$ if $v \in W^{1}\left(\mathbb{R}^{2}\right)$ is a solution of

$$
\begin{cases}\Delta v=0 & \text { in } \mathbb{R}^{2} \backslash \bar{B}_{1}, \\ \operatorname{div}(a \nabla v)+k^{2} \sigma v=0 & \text { in } B_{1}, \\ \left.\frac{\partial v}{\partial \eta}\right|_{\text {ext }}=\left.a \nabla v \cdot \eta\right|_{\text {int }} & \text { on } \partial B_{1} .\end{cases}
$$

Since $v \in W^{1}\left(\mathbb{R}^{2}\right)$, it follows from (3.19) that (see, e.g., [17] or [19, prop. 2])

$$
\left.\int_{\partial B_{1}} \frac{\partial v}{\partial \eta}\right|_{\text {ext }}=0
$$

(this identity is the key to the proof) and

$$
\int_{\mathbb{R}^{2} \backslash B_{1}}|\nabla v|^{2}+\int_{B_{1}}\langle a \nabla v, \nabla v\rangle-\int_{B_{1}} k^{2} \sigma|v|^{2}=0 .
$$

Integrating the equation $\operatorname{div}(a \nabla v)+k^{2} \sigma v=0$ on $B_{1}$ and using (3.20), we have

$$
\int_{B_{1}} \sigma v=0
$$

which implies, by a standard compactness argument,

$$
\|\nabla v\|_{L^{2}\left(B_{1}\right)} \geq C\|v\|_{L^{2}\left(B_{1}\right)}
$$

for some positive constant $C$ depending only on $c_{1}$ and $c_{2}$. Combining (3.21) and (3.23) yields

$$
\int_{\mathbb{R}^{2} \backslash B_{1}}|\nabla v|^{2}+\int_{B_{1}}\langle a \nabla v, \nabla v\rangle=0
$$


if $0<k<k_{0}$ for some $k_{0}>0$. This implies that $v$ is constant. Hence $v=0$ by (3.22).

\section{Proof of Proposition 1.11}

Define $U_{\varepsilon}=u_{\varepsilon}(\varepsilon x)$ where $u_{\varepsilon}=u_{c} \circ F_{\varepsilon}$. Then $U_{\varepsilon}$ is the solution of

$$
\begin{cases}\Delta U_{\varepsilon}+\varepsilon^{2} k^{2} U_{\varepsilon}=0 & \text { in } \mathbb{R}^{3} \backslash \bar{B}_{1}, \\ \operatorname{div}\left(a \nabla U_{\varepsilon}\right)+k^{2} \sigma U_{\varepsilon}=\mathbf{e} & \text { in } B_{1}, \\ \left.\frac{\partial U_{\varepsilon}}{\partial \eta}\right|_{\text {ext }}-\left.\frac{1}{\varepsilon} a \nabla U_{\varepsilon} \cdot \eta\right|_{\text {int }}=0 & \text { on } \partial B_{1}, \\ U_{\varepsilon} \text { satisfies the outgoing condition. } & \end{cases}
$$

PROOF OF PART (i). It suffices to prove

$$
\liminf _{\varepsilon \rightarrow 0} \varepsilon\left\|U_{\varepsilon}\right\|_{H^{1 / 2}\left(\partial B_{1}\right)}>0,
$$

since $u_{c}=U_{\varepsilon}$ in $B_{1}$. We will prove (4.1) by contradiction. Suppose that there exists $\varepsilon_{n} \rightarrow 0$ such that

$$
\lim _{n \rightarrow \infty} \varepsilon_{n}\left\|U_{n}\right\|_{H^{1 / 2}\left(\partial B_{1}\right)}=0,
$$

where $U_{n}$ is the solution of

$$
\begin{cases}\Delta U_{n}+\varepsilon_{n}^{2} k^{2} U_{n}=0 & \text { in } \mathbb{R}^{3} \backslash \bar{B}_{1}, \\ \operatorname{div}\left(a \nabla U_{n}\right)+k^{2} \sigma U_{n}=\mathbf{e} & \text { in } B_{1}, \\ \left.\frac{\partial U_{n}}{\partial \eta}\right|_{\text {ext }}-\left.\frac{1}{\varepsilon_{n}} a \nabla U_{n} \cdot \eta\right|_{\text {int }}=0 & \text { on } \partial B_{1}, \\ U_{n} \text { satisfies the outgoing condition. } & \end{cases}
$$

By Lemma 2.2, we have

$$
\lim _{n \rightarrow \infty} \varepsilon_{n}\left\|U_{n}\right\|_{H^{1}\left(B_{r} \backslash \bar{B}_{1}\right)}=0 \quad \forall r>1,
$$

which implies, since $\Delta U_{n}+\varepsilon_{n}^{2} k^{2} U_{n}=0$ in $\mathbb{R}^{3} \backslash \bar{B}_{1}$,

$$
\lim _{n \rightarrow \infty} \varepsilon_{n}\left\|\left.\frac{\partial U_{n}}{\partial \eta}\right|_{\text {ext }}\right\| H_{H^{-1 / 2}\left(\partial B_{1}\right)}=0 .
$$

Hence, since $\left.\frac{\partial U_{n}}{\partial \eta}\right|_{\text {ext }}-\left.\frac{1}{\varepsilon_{n}} a \nabla U_{n} \cdot \eta\right|_{\text {int }}=0$ on $\partial B_{1}$, we have

$$
\lim _{n \rightarrow \infty}\left\|\left.a \nabla U_{n} \cdot \eta\right|_{\text {int }}\right\|_{H^{-1 / 2}\left(\partial B_{1}\right)}=0 .
$$

Multiplying the equation $\operatorname{div}\left(a \nabla U_{n}\right)+k^{2} \sigma U_{n}=\mathbf{e}$ by $\overline{\mathbf{e}}$, integrating the expression obtained on $B_{1}$, and using the fact that $\mathbf{e} \in M$, we have

$$
\int_{\partial B_{1}}\left(a \nabla U_{n} \cdot \eta\right) \overline{\mathbf{e}}=\int_{B_{1}}|\mathbf{e}|^{2}=1 .
$$

Combining (4.2) and (4.3), we have a contradiction. 
ProOf OF PART (ii). Since $\mathbf{e}$ is radial, $a=\lambda_{1} I$, and $\sigma=\lambda_{2}$ for some positive constants $\lambda_{1}$ and $\lambda_{2}$, it follows that $U_{\varepsilon}$ is radial. This implies $U_{\varepsilon}(x)=c_{\varepsilon} \frac{e^{i k \varepsilon|x|}}{|x|}$ if $|x|>1$ for some positive constant $c_{\varepsilon}$. Hence the conclusion of part (ii) follows from (4.1) and the fact that $u_{c}(x)=U_{\varepsilon}(x / \varepsilon)$ for $x \in B_{4} \backslash B_{2}$.

\section{Proof of Proposition 1.12}

Define $U_{\varepsilon}=u_{\varepsilon}(\varepsilon x)$ where $u_{\varepsilon}=u_{c} \circ F_{\varepsilon}$. Then $U_{\varepsilon}$ is the solution of

$$
\begin{cases}\Delta U_{\varepsilon}+\varepsilon^{2} k^{2} U_{\varepsilon}=0 & \text { in } \mathbb{R}^{2} \backslash \bar{B}_{1}, \\ \operatorname{div}\left(a \nabla U_{\varepsilon}\right)+k^{2} \sigma U_{\varepsilon}=\mathbf{e} & \text { in } B_{1}, \\ \left.\frac{\partial U_{\varepsilon}}{\partial \eta}\right|_{\text {ext }}-\left.a \nabla U_{\varepsilon} \cdot \eta\right|_{\text {int }}=0 & \text { on } \partial B_{1}, \\ U_{\varepsilon} \text { satisfies the outgoing condition. } & \end{cases}
$$

PROOF OF PART (i). It suffices to prove

$$
\liminf _{\varepsilon \rightarrow 0}\left\|U_{\varepsilon}\right\|_{H^{1}\left(B_{1}\right)}=\infty,
$$

since $u_{c}=U_{\varepsilon}$ in $B_{1}$. We will prove (5.1) by contradiction. Suppose that there exists $\varepsilon_{n} \rightarrow 0$ such that

$$
\sup _{n \in \mathbb{N}}\left\|U_{n}\right\|_{H^{1}\left(B_{1}\right)}<+\infty,
$$

where $U_{n}$ is the solution of

$$
\begin{cases}\Delta U_{n}+\varepsilon_{n}^{2} k^{2} U_{n}=0 & \text { in } \mathbb{R}^{2} \backslash \bar{B}_{1}, \\ \operatorname{div}\left(a \nabla U_{n}\right)+k^{2} \sigma U_{n}=\mathbf{e} & \text { in } B_{1}, \\ \left.\frac{\partial U_{n}}{\partial \eta}\right|_{\text {ext }}-\left.a \nabla U_{n} \cdot \eta\right|_{\text {int }}=0 & \text { on } \partial B_{1}, \\ U_{n} \text { satisfies the outgoing condition. } & \end{cases}
$$

By Lemma 3.2, we have

$$
\sup _{n \in \mathbb{N}}\left\|U_{n}\right\|_{H^{1}\left(B_{r} \backslash \bar{B}_{1}\right)}<+\infty \quad \forall r>1 .
$$

It follows from (5.2) that

$$
\sup _{n \in \mathbb{N}}\left\|U_{n}\right\|_{H^{1}\left(B_{r}\right)}<+\infty \quad \forall r>1 .
$$

Applying Lemma 3.2, we may assume that $U_{n} \rightarrow U$ in $H_{\text {loc }}^{1}\left(\mathbb{R}^{2}\right)$ for some $U \in$ $W^{1}\left(\mathbb{R}^{2}\right)$ that satisfies

$$
\begin{cases}\Delta U=0 & \text { in } \mathbb{R}^{2} \backslash \bar{B}_{1}, \\ \operatorname{div}(a \nabla U)+k^{2} \sigma U=\mathbf{e} & \text { in } B_{1}, \\ \left.\frac{\partial U}{\partial \eta}\right|_{\mathrm{ext}}-\left.a \nabla U \cdot \eta\right|_{\mathrm{int}}=0 & \text { on } \partial B_{1} .\end{cases}
$$

However, system (5.3) has no solution in $W^{1}\left(\mathbb{R}^{2}\right)$ since $\mathbf{e} \in N$. We have a contradiction. 
ProOF OF PART (ii). For notational ease, we will assume that $\lambda_{1}=1$ and $\lambda_{2}=\lambda$. Since $\mathbf{e}$ is radial, $a=I$, and $\sigma=\lambda, U_{\varepsilon}$ is radial. Thus $U_{\varepsilon}$ can be written under the form

$$
U_{\varepsilon}=c_{\varepsilon} H_{0}^{(1)}(\varepsilon k r) \text { for } r=|x| \geq 1 .
$$

This implies

$$
\frac{\partial U_{\varepsilon}}{\partial \eta}=c_{\varepsilon} \varepsilon k \partial_{r} H_{0}^{(1)}(\varepsilon k) \quad \text { for } r=|x|=1 .
$$

Since $\mathbf{e}$ is radial, $\mathbf{e} \in W^{1}\left(\mathbb{R}^{2} \backslash \bar{B}_{1}\right)$, and $\Delta \mathbf{e}=0$ in $\mathbb{R}^{2} \backslash \bar{B}_{1}$, it follows that $\mathbf{e}$ is constant in $\mathbb{R}^{2} \backslash B_{1}$. Hence

$$
\frac{\partial \mathbf{e}}{\partial \eta}=0 \quad \text { on } \partial B_{1}
$$

We have

$$
\Delta U_{\varepsilon}+k^{2} \lambda U_{\varepsilon}=\mathbf{e} \text { in } B_{1} \text { and } \Delta \mathbf{e}+k^{2} \lambda \mathbf{e}=0 \text { in } B_{1} .
$$

Multiplying the first equation by $\overline{\mathbf{e}}$, the second equation by $\bar{U}_{\varepsilon}$, and integrating the obtained expressions on $B_{1}$, we have

$$
\int_{B_{1}} \nabla U_{\varepsilon} \nabla \overline{\mathbf{e}}-k^{2} \lambda U_{\varepsilon} \overline{\mathbf{e}}=-\int_{B_{1}}|\mathbf{e}|^{2}+\int_{\partial B_{1}} \frac{\partial U_{\varepsilon}}{\partial \eta} \overline{\mathbf{e}}
$$

and

$$
\int_{B_{1}} \nabla \mathbf{e} \nabla \bar{U}_{\varepsilon}-k^{2} \lambda \mathbf{e} \bar{U}_{\varepsilon}=\int_{\partial B_{1}} \frac{\partial \mathbf{e}}{\partial \eta} \bar{U}_{\varepsilon}=0
$$

(in the last equality, we used (5.5)). This implies

$$
1=\int_{B_{1}}|\mathbf{e}|^{2}=\int_{\partial B_{1}} \frac{\partial U_{\varepsilon}}{\partial \eta} \overline{\mathbf{e}} .
$$

It follows from (5.4) that

$$
\int_{\partial B_{1}} \overline{\mathbf{e}} c_{\varepsilon} \varepsilon k \partial_{r} H_{0}^{(1)}(\varepsilon k)=1 .
$$

We recall that (see, e.g., [3, chap. 3])

$$
\lim _{r \rightarrow 0} \frac{r d H_{0}^{(1)}(r)}{d r}=-\frac{2}{i \pi},
$$

which yields

$$
\left|c_{\varepsilon}\right| \geq c
$$

for some positive constant $c$. Hence

$$
\left|U_{\varepsilon}(x / \varepsilon)\right| \geq c\left|H_{0}^{(1)}(k|x|)\right| \text { for } 2 \leq|x| \leq 4 .
$$

Since $u_{c}(x)=U_{\varepsilon}(x / \varepsilon)$ for $x \in B_{4} \backslash B_{2}$, the conclusion of part (ii) follows. 


\section{Proof of Proposition 1.13}

This section is devoted to the proof of Proposition 1.13. It suffices to prove the following proposition:

Proposition 6.1. Let $d=2,3, k>0$, and $\sigma_{0}>0$ be such that $j_{0}^{\prime}\left(k \sigma_{0}\right)=0$ if $d=3$ and $J_{0}^{\prime}\left(k \sigma_{0}\right)=0$ if $d=2$. Then there exists a family of positive numbers $\left(\sigma_{\varepsilon}\right)$ such that

$$
\begin{cases}0<\liminf _{\varepsilon \rightarrow 0} \varepsilon^{-1}\left|\sigma_{\varepsilon}-\sigma_{0}\right| \leq \limsup _{\varepsilon \rightarrow 0} \varepsilon^{-1}\left|\sigma_{\varepsilon}-\sigma_{0}\right|<+\infty & \text { if } d=3, \\ 0<\liminf _{\varepsilon \rightarrow 0}|\ln \varepsilon|\left|\sigma_{\varepsilon}-\sigma_{0}\right| \leq \limsup _{\varepsilon \rightarrow 0}|\ln \varepsilon|\left|\sigma_{\varepsilon}-\sigma_{0}\right|<+\infty & \text { if } d=2,\end{cases}
$$

and

$$
\left\|u_{c, s}\right\|_{L^{2}\left(B_{4} \backslash B_{2}\right)} \geq \begin{cases}\left\|h_{0}\right\|_{L^{2}\left(B_{4} \backslash B_{2}\right)} & \text { if } d=3, \\ \left\|H_{0}\right\|_{L^{2}\left(B_{4} \backslash B_{2}\right)} & \text { if } d=2 .\end{cases}
$$

Here $u_{c, s} \in H_{\mathrm{loc}}^{1}\left(\mathbb{R}^{d}\right)$ is such that $u_{c, s}$ satisfies the outgoing condition and if $u_{c}:=u_{c, s}+u_{c, i}$ with

$$
u_{c, i}= \begin{cases}j_{0}(k|x|) & \text { if } d=3, \\ J_{0}(k|x|) & \text { if } d=2,\end{cases}
$$

then $u_{c}$ is a solution of the equation

$$
\operatorname{div}\left(A_{c} \nabla u_{c}\right)+k^{2} \Sigma_{c} u_{c}=0,
$$

where $\left(A_{c}, \Sigma_{c}\right)$ is defined in (1.5) with $a=I$ and $\sigma=\sigma_{\varepsilon}$.

Hereafter $h_{0}$ denotes the spherical Hankel function of the first kind of order 0 , $j_{0}=\Re\left(h_{0}\right)$, and $y_{0}=\Im\left(h_{0}\right)$, and $H_{0}$ denotes the Hankel function of the first kind of order $0, J_{0}=\Re\left(H_{0}\right)$, and $Y_{0}=\Im\left(H_{0}\right)$.

Proof. Set $u_{\varepsilon}=u_{c} \circ F_{\varepsilon}$ and $u_{\varepsilon, s}=u_{\varepsilon}-u_{c, i}$ where $F_{\varepsilon}$ is given in (1.1). According to Proposition $1.15, u_{\varepsilon}=u_{c}, u_{\varepsilon, s}=u_{c, s}$ in $\mathbb{R}^{d} \backslash B_{2}, u_{\varepsilon, s}$ satisfies the outgoing condition, and $u_{\varepsilon}$ is the solution of the equation

$$
\operatorname{div}\left(A_{\varepsilon} \nabla u_{\varepsilon}\right)+k^{2} \Sigma_{\varepsilon} u_{\varepsilon}=0
$$

where

$$
A_{\varepsilon}, \Sigma_{\varepsilon}= \begin{cases}I, 1 & \text { if } x \in \mathbb{R}^{d} \backslash B_{\varepsilon} \\ \frac{I}{\varepsilon^{d-2}}, \frac{\sigma_{\varepsilon}}{\varepsilon^{d}} & \text { if } x \in B_{\varepsilon} .\end{cases}
$$

Define $U_{\varepsilon}(x)=u_{\varepsilon}(\varepsilon x)$ and $U_{\varepsilon, s}(x)=u_{\varepsilon, s}(\varepsilon x)=U_{\varepsilon}(x)-u_{c, i}(\varepsilon x)$ for $x \in \mathbb{R}^{d}$. Then $U_{\varepsilon, s}(x)=u_{c, s}(\varepsilon x)$ for $|x|>2 / \varepsilon, U_{\varepsilon}$ satisfies the equation

$$
\operatorname{div}\left(\widetilde{A}_{\varepsilon} \nabla U_{\varepsilon}\right)+k^{2} \widetilde{\Sigma}_{\varepsilon} U_{\varepsilon}=0,
$$

and $U_{\varepsilon, s}$ satisfies the outgoing condition. Here

$$
\tilde{A}_{\varepsilon}, \widetilde{\Sigma}_{\varepsilon}= \begin{cases}I, \varepsilon^{2} & \text { if } x \in \mathbb{R}^{d} \backslash B_{1}, \\ \frac{I}{\varepsilon^{d-2}}, \frac{\sigma_{\varepsilon}}{\varepsilon^{d-2}} & \text { if } x \in B_{1}\end{cases}
$$


Step $1 . d=3$. It is clear that

$$
U_{\varepsilon, i}=j_{0}(k \varepsilon|x|), \quad U_{\varepsilon, s}=\alpha_{0} h_{0}(k \varepsilon|x|) \quad \text { for }|x|>1,
$$

and

$$
U_{\varepsilon, t}=\beta_{0} j_{0}\left(k_{\varepsilon}|x|\right) \text { for }|x|<1,
$$

where $k_{\varepsilon}=k \sigma_{\varepsilon}$ and $U_{\varepsilon, t}:=U_{\varepsilon, s}+U_{\varepsilon, i}=U_{\varepsilon}$ in $B_{1}$ for some $\alpha_{0}, \beta_{0}$. Using the transmission conditions, namely,

$$
\begin{cases}U_{\varepsilon, s}+U_{\varepsilon, i}=U_{\varepsilon, t} & \text { on } \partial B_{1} \\ \frac{\partial U_{\varepsilon, s}}{\partial r}+\frac{\partial U_{\varepsilon, i}}{\partial r}=\frac{1}{\varepsilon} \frac{\partial U_{\varepsilon, t}}{\partial r} & \text { on } \partial B_{1}\end{cases}
$$

we have

$$
\begin{cases}\alpha_{0} h_{0}(k \varepsilon)+j_{0}(k \varepsilon)=\beta_{0} j_{0}\left(k_{\varepsilon}\right) & \text { on } \partial B_{1}, \\ \alpha_{0} k \varepsilon h_{0}^{\prime}(\varepsilon)+k \varepsilon j_{0}^{\prime}(\varepsilon)=\frac{1}{\varepsilon} k_{\varepsilon} \beta_{0} j_{0}^{\prime}\left(k_{\varepsilon}\right) & \text { on } \partial B_{1} .\end{cases}
$$

It follows that

$$
\alpha_{0}=-\frac{k \varepsilon j_{0}^{\prime}(k \varepsilon) j_{0}\left(k_{\varepsilon}\right)-\frac{1}{\varepsilon} k_{\varepsilon} j_{0}(k \varepsilon) j_{0}^{\prime}\left(k_{\varepsilon}\right)}{k \varepsilon h_{0}^{\prime}(k \varepsilon) j_{0}\left(k_{\varepsilon}\right)-\frac{1}{\varepsilon} k_{\varepsilon} h_{0}(k \varepsilon) j_{0}^{\prime}\left(k_{\varepsilon}\right)} .
$$

Since $y_{0}(t)=\cos t / t$, we have

$$
k \varepsilon^{2} \frac{y_{0}^{\prime}(k \varepsilon)}{y_{0}(k \varepsilon)}=k \varepsilon^{2}\left(-\frac{1}{k \varepsilon}-\frac{\sin (k \varepsilon)}{\cos (k \varepsilon)}\right)=-\varepsilon-k \varepsilon^{2} \frac{\sin (k \varepsilon)}{\cos (k \varepsilon)} .
$$

Let $\sigma_{\varepsilon}$ be such that $k_{\varepsilon}=k \sigma_{\varepsilon}$ converges to $k \sigma_{0}$ and

$$
\frac{j_{0}^{\prime}\left(k_{\varepsilon}\right)}{j_{0}\left(k_{\varepsilon}\right)}=-\varepsilon-k \varepsilon^{2} \frac{\sin (k \varepsilon)}{\cos (k \varepsilon)} \text {. }
$$

Then the complex part in the denominator of the right-hand side of (6.1) equals 0 . On the other hand, the real part in the denominator of the right-hand side of (6.1) equals the numerator of the right-hand side of (6.1). Hence it follows from (6.1) that

$$
\alpha_{0}=-1,
$$

implying the conclusion in the three-dimensional case since $u_{c, s}(x)=U_{\varepsilon, s}(x / \varepsilon)$ for $|x|>2$.

Step 2. $d=2$. It is clear that

$$
U_{\varepsilon, i}=J_{0}(k \varepsilon|x|), \quad U_{\varepsilon, s}=\alpha_{0} H_{0}(k \varepsilon|x|) \quad \text { for }|x|>1,
$$

and

$$
U_{\varepsilon, t}=\beta_{0} J_{0}\left(k_{\varepsilon}|x|\right) \quad \text { for }|x|<1,
$$

where $k_{\varepsilon}=k \sigma_{\varepsilon}, U_{\varepsilon, t}:=U_{\varepsilon, s}+U_{\varepsilon, i}=U_{\varepsilon}$ in $B_{1}$, for some $\alpha_{0}, \beta_{0}$. Using the transmission conditions, namely,

$$
\begin{cases}U_{\varepsilon, s}+U_{\varepsilon, i}=U_{\varepsilon, t} & \text { on } \partial B_{1}, \\ \frac{\partial U_{\varepsilon, s}}{\partial r}+\frac{\partial U_{\varepsilon, i}}{\partial r}=\frac{\partial U_{\varepsilon, t}}{\partial r} & \text { on } \partial B_{1}\end{cases}
$$


we have

$$
\begin{cases}\alpha_{0} H_{0}(k \varepsilon)+J_{0}(k \varepsilon)=\beta_{0} J_{0}\left(k_{\varepsilon}\right) & \text { on } \partial B_{1}, \\ \alpha_{0} k \varepsilon H_{0}^{\prime}(k \varepsilon)+k \varepsilon J_{0}^{\prime}(k \varepsilon)=k_{\varepsilon} \beta_{0} J_{0}^{\prime}\left(k_{\varepsilon}\right) & \text { on } \partial B_{1} .\end{cases}
$$

Thus it follows that

$$
\alpha_{0}=-\frac{k \varepsilon J_{0}^{\prime}(k \varepsilon) J_{0}\left(k_{\varepsilon}\right)-k_{\varepsilon} J_{0}(k \varepsilon) J_{0}^{\prime}\left(k_{\varepsilon}\right)}{k \varepsilon H_{0}^{\prime}(k \varepsilon) J_{0}\left(k_{\varepsilon}\right)-k_{\varepsilon} H_{0}(k \varepsilon) J_{0}^{\prime}\left(k_{\varepsilon}\right)} .
$$

Since $Y_{0}(t)=\frac{2}{\pi} \ln (t / 2)$, we have

$$
k \varepsilon \frac{Y_{0}^{\prime}(k \varepsilon)}{Y_{0}(k \varepsilon)}=\frac{1}{\ln (k \varepsilon / 2)} .
$$

Let $\sigma_{\varepsilon}$ be such that $k_{\varepsilon}=k \sigma_{\varepsilon} \rightarrow k \sigma_{0}$ and

$$
\frac{k_{\varepsilon} J_{0}^{\prime}\left(k_{\varepsilon}\right)}{J_{0}\left(k_{\varepsilon}\right)}=\frac{1}{\ln (k \varepsilon / 2)} .
$$

Then as in the three-dimensional case, $\alpha_{0}=-1$, and the conclusion in the twodimensional case follows from the fact that $u_{c, s}(x)=U_{\varepsilon, s}(x / \varepsilon)$ for $|x|>2$.

Acknowledgment. The author thanks Bob Kohn for stimulating discussions and useful comments on the manuscript.

\section{Bibliography}

[1] Brezis, H. Analyse fonctionnelle. Théorie et applications. Collection Mathématiques Appliquées pour la Maîtrise. Dunod, Paris, 2002.

[2] Cai, W.; Chettiar, U. K.; Kildishev, A. V.; Shalaev, V. M. Optical cloaking with metamaterials. Nature Photonics 1 (2007), 224-227. Available at: http://www. nature.com/nphoton/ journal/v1/n4/abs/nphoton.2007.28.html.

[3] Colton, D.; Kress, R. Inverse acoustic and electromagnetic scattering theory. 2nd ed. Applied Mathematical Sciences, 93. Springer, Berlin, 1998.

[4] Cummer, S. A.; Popa, B. I.; Schurig, D.; Smith, D. R.; Pendry, J.; Rahm, M.; Starr, A. Scattering theory derivation of $3 d$ acoustic cloaking shell. Phys. Rev. Lett. 100 (2008), no. 2, 024301, 4 pp.

[5] Greenleaf, A.; Kurylev, Y.; Lassas, M.; Uhlmann, G. Full-wave invisibility of active devices at all frequencies. Comm. Math. Phys. 275 (2007), no. 3, 749-789. doi:10.1007/s00220-0070311-6.

[6] Greenleaf, A.; Kurylev, Y.; Lassas, M.; Uhlmann, G. Improvement of cylindrical cloaking with the SHS lining. Optics Express 15 (2007), no. 20, 12717-12734. doi:10.1364/OE.15.012717.

[7] Greenleaf, A.; Kurylev, Y.; Lassas, M.; Uhlmann, G. Isotropic transformation optics: approximate acoustic and quantum cloaking. New J. Phys. 10 (2008), 115024-115051. doi:10.1088/ 1367-2630/10/11/115024.

[8] Greenleaf, A.; Kurylev, Y.; Lassas, M.; Uhlmann, G. Invisibility and inverse problems. Bull. Amer. Math. Soc. (N.S.) 46 (2009), no. 1, 55-97.

[9] Greenleaf, A.; Kurylev, Y.; Lassas, M.; Uhlmann, G. Cloaking a sensor via transformation optics. Phys. Rev. E 83 (2011), 016603.

[10] Greenleaf, A.; Lassas, M.; Uhlmann, G. On nonuniqueness for Calderon's inverse problem. Math. Res. Lett. 10 (2003), no. 5-6, 685-693. 
[11] Kohn, R. V.; Onofrei, D.; Vogelius, M. S.; Weinstein, M. I. Cloaking via change of variables for the Helmholtz equation. Comm. Pure Appl. Math. 63 (2010), no. 8, 973-1016.

[12] Kohn, R. V.; Shen, H.; Vogelius, M. S.; Weinstein, M. I. Cloaking via change of variables in electric impedance tomography. Inverse Problems 24 (2008), no. 1, 015016, 21 pp.

[13] Lassas, M.; Zhou, T. Two dimensional invisibility cloaking for Helmholtz equation and nonlocal boundary conditions. Math. Res. Lett. 18 (2011), no. 3, 473-488.

[14] Lax, P. D. Functional analysis. Pure and Applied Mathematics (New York). Wiley-Interscience, New York, 2002.

[15] Leonhardt, U. Optical conformal mapping. Science 312 (2006), no. 5781, 1777-1780.

[16] Liu, H. Virtual reshaping and invisibility in obstacle scattering. Inverse Problems 25 (2009), no. 4, 045006, $16 \mathrm{pp}$.

[17] Nédélec, J.-C. Acoustic and electromagnetic equations. Integral representations for harmonic problems. Applied Mathematical Sciences, 144. Springer, New York, 2001.

[18] Nguyen, H-M. Cloaking via change of variables for the Helmholtz equation in the whole space. Comm. Pure Appl. Math. 63 (2010), no. 11, 1505-1524.

[19] Nguyen, H-M.; Vogelius, M. S. A representation formula for the voltage perturbations caused by diametrically small conductivity inhomogeneities. Proof of uniform validity. Ann. Inst. H. Poincaré Anal. Non Linéaire 26 (2009), no. 6, 2283-2315.

[20] Nguyen, H-M.; Vogelius, M. S. Full range scattering estimates and their application to cloaking. Preprint, 2010. Submitted to Arch. Ration. Mech. Anal. Available at: http://www . cims.nyu.edu/ hoaiminh/download/publications/H-M.Nguyen_Publication_ FullRangeScatteringEstimatesandtheirApplicationtoCloaking.pdf.

[21] Nguyen, H-M.; Vogelius, M. S. Approximate cloaking for the full wave equation via change of variables. Preprint, 2011. Submitted to SIAM J. Math. Anal. Available at: http: / / www . math . rutgers.edu/ vogelius/papers/37pp.pdf.

[22] Pendry, J. B.; Schurig, D.; Smith, D. R. Controlling electromagnetic fields. Science 312 (2006), no. 5781, 1780-1782.

[23] Ruan, Z.; Yan, M.; Neff, C. W.; Qiu, M. Ideal cylindrical cloak: perfect but sensitive to tiny perturbations. Phys. Rev. Lett. 99 (2007), no. 11, 113903.

[24] Schurig, D.; Mock, J. J.; Justice, B. J.; Cummer, S. A.; Pendry, J. B.; Starr, A. F.; Smith, D. R. Metamaterial electromagnetic cloak at microwave frequencies. Science 314 (2006), no. 5801, 977-980.

[25] Vogelius, M. S.; Volkov, D. Asymptotic formulas for perturbations in the electromagnetic fields due to the presence of inhomogeneities of small diameter. M2AN Math. Model. Numer. Anal. 34 (2000), no. 4, 723-748.

[26] Weder, R. The boundary conditions for point transformed electromagnetic invisibility cloaks. J. Phys. A 41 (2008), no. 41, 415401, 17 pp.

[27] Weder, R. A rigorous analysis of high-order electromagnetic invisibility cloaks. J. Phys. A 41 (2008), no. 6, 065207, 21 pp.

[28] Wood, B. Metamaterials and invisibility. C. R. Phys. 10 (2009), no. 5, 379-390.

[29] Yan, M.; Ruan, Z.; Qiu, M. Cylindrical invisibility cloak with simplified material parameters is inherently visible. Phys. Rev. Lett. 99 (2007), 233901, 4 pp. Available at: http: / / i ink . aps . org/doi/10.1103/PhysRevLett.99.233901. 
HoAi-Minh NGUYEN

University of Minnesota

School of Mathematics

206 Church St. SE

Minneapolis, MN, 55455

E-mail: hmnguyen@umn .edu

Received October 2010.

Revised August 2011. 